\title{
Sample-Optimal and Efficient Learning of Tree Ising Models
}

\author{
Constantinos Daskalakis \\ EECS and CSAIL, MIT, USA
}

\author{
Qinxuan Pan \\ Formerly EECS and CSAIL, MIT, USA
}

\begin{abstract}
We show that $n$-variable tree-structured Ising models can be learned computationally-efficiently to within total variation distance $\epsilon$ from an optimal $O\left(n \ln n / \epsilon^{2}\right)$ samples, where $O(\cdot)$ hides an absolute constant which, importantly, does not depend on the model being learned-neither its tree nor the magnitude of its edge strengths, on which we place no assumptions. Our guarantees hold, in fact, for the celebrated Chow-Liu algorithm [1968], using the plug-in estimator for estimating mutual information. While this (or any other) algorithm may fail to identify the structure of the underlying model correctly from a finite sample, we show that it will still learn a tree-structured model that is $\epsilon$-close to the true one in total variation distance, a guarantee called "proper learning."

Our guarantees do not follow from known results for the ChowLiu algorithm and the ensuing literature on learning graphical models, including the very recent renaissance of algorithms on this learning challenge, which only yield asymptotic consistency results, or sample-suboptimal and/or time-inefficient algorithms, unless further assumptions are placed on the model, such as bounds on the "strengths" of the model's edges. While we establish guarantees for a widely known and simple algorithm, the analysis that this algorithm succeeds and is sample-optimal is quite complex, requiring a hierarchical classification of the edges into layers with different reconstruction guarantees, depending on their strength, combined with delicate uses of the subadditivity of the squared Hellinger distance over graphical models to control the error accumulation.
\end{abstract}

\section{CCS CONCEPTS}

- Theory of computation $\rightarrow$ Sample complexity and generalization bounds; • Mathematics of computing $\rightarrow$ Bayesian networks; Density estimation.

\section{KEYWORDS}

Tree-Structured Model, Bayesian Network, Ising Model, Markov Random Field, Chow-Liu Algorithm, Subadditivity, Hellinger Distance, Estimation, Sample Complexity

\section{ACM Reference Format:}

Constantinos Daskalakis and Qinxuan Pan. 2021. Sample-Optimal and Efficient Learning of Tree Ising Models. In Proceedings of the 53rd Annual ACM SIGACT Symposium on Theory of Computing (STOC '21), fune 21-25, 2021, Virtual, Italy. ACM, New York, NY, USA, 14 pages. https: //doi.org/10.1145/3406325.3451006

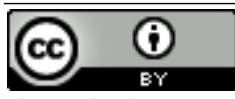

This work is licensed under a Creative Commons Attribution International 4.0 License. STOC '21, fune 21-25, 2021, Virtual, Italy

(C) 2021 Copyright held by the owner/author(s).

ACM ISBN 978-1-4503-8053-9/21/06.

https://doi.org/10.1145/3406325.3451006

\section{INTRODUCTION}

Markov Random Fields (MRFs) and Bayesian Networks (Bayesnets) are popular frameworks for representing high-dimensional distributions graphically, allowing the explicit specification of conditional independence properties that a high-dimensional distribution may satisfy. These properties can be exploited to decrease the sample and computational requirements to learn the distribution, as well as to perform inference once the distribution has been learned. A particularly simple special case of both frameworks occurs when the underlying graph is a tree. In this case, many basic inference tasks that are computationally intractable even to approximate in general, e.g. computing marginals or the mode of the distribution, can be carried out exactly by simple, linear-time algorithms, such as the popular sum-product and max-product algorithms. For an introduction to MRFs and Bayesnets, their uses, and associated algorithms see, e.g., $[12,14,20]$.

The attractiveness of these graphical models and their widespread use in application domains have motivated a vast literature on learning them from samples; see e.g. the references above and $[1-3,8-10,13,15-19,21]$. Even when the model to be learned is tree-structured, however, these methods only yield asymptotic consistency results, or sample-suboptimal and/or time-inefficient algorithms, unless further assumptions are placed on the distribution. Indeed, recent works on efficiently learning Ising models (and more general MRFs) only provide guarantees that the learned distribution is close (under some probabilistic distance) to the one being learned, assuming a bound on the total "strength" of the edges (resp. hyperedges) adjacent to each node. Under even stronger assumptions, some of these works also identify the graphical structure of the Ising model (resp. MRF).

Our goal in this paper is to study whether a tree-structured Ising model (equivalently a tree-structured binary-alphabet Bayesnet or a tree-structured binary-alphabet MRF) can be learned without making any assumptions about the distribution. More precisely, we study the following problem.

Learning Problem: Given samples from an arbitrary n-variable tree-structued Ising model $\mathrm{P}$, learn some $n$-variable distribution $\mathrm{Q}$ that is within some desired total variation distance $\epsilon>0$ from $\mathrm{P}$, using a number of samples and total computation time that scale polynomially in $n$ and $1 / \epsilon$, and are independent of any property of $\mathrm{P}$.

We will additionally insist on what is called proper learning, i.e. aim to compute a tree-structured Ising model $\mathrm{Q}$ such that $d_{\mathrm{TV}}(\mathrm{P}, \mathrm{Q}) \leq \epsilon$. We want to identify a tree-structured Ising model $Q$ as we want to maintain the computational benefits of tree-structured models when performing inference tasks on the learned Q. And, of course, we want that $Q$ is close to the true model $P$ in total variation distance so that the inferences on $\mathrm{Q}$ are approximately correct. We show that the celebrated Chow-Liu algorithm [2] attains these guarantees from an optimal number of samples. 
Theorem 1 (Main Theorem-Restated as Theorem 22 in SecTION 4). For $n \in \mathbb{N}, \epsilon, \gamma \in(0,1]$, given $O\left(\frac{n(\ln n+\ln 1 / \gamma)}{\epsilon^{2}}\right)$ samples from an arbitrary $n$-variable binary-alphabet Bayesnet $\mathrm{P}$ defined on an unknown tree, the Chow-Liu algorithm with the plug-in estimator for estimating mutual information (namely Algorithm 1), learns an $n$-variable tree-structured Bayesnet $\mathrm{Q}$ on a (possibly different) tree such that $d_{\mathrm{TV}}(\mathrm{P}, \mathrm{Q}) \leq \epsilon$ with probability at least $1-\gamma$. The running time of the algorithm is polynomial, and is dominated by the time to compute the mutual information between all pairs of variables on the empirical distribution defined by the samples, and running a Maximum Spanning tree algorithm. Finally, the number of samples is optimal up to a constant factor. Importantly, the $O(\cdot)$ in our sample complexity is hiding an absolute constant which does not depend on the model being learned-neither its tree nor the magnitude of the edge strengths.

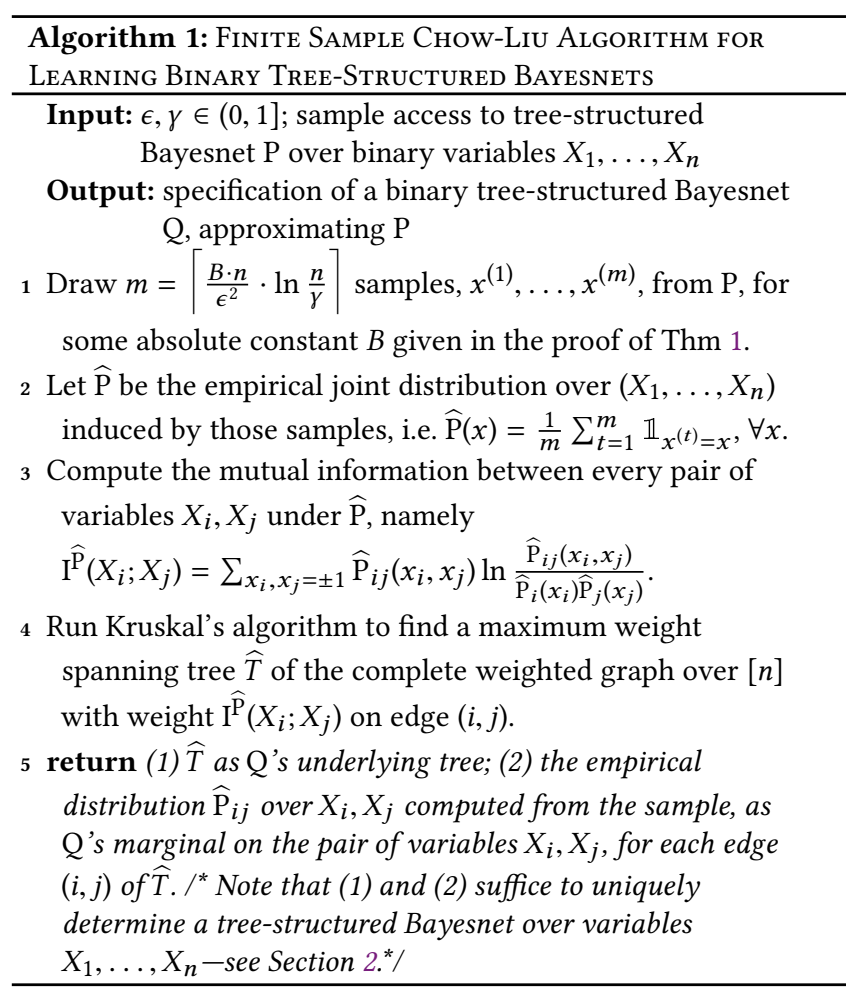

We note that it is well-understood that the celebrated Chow-Liu algorithm [2] can be used to obtain an asymptotically consistent estimator of tree-structured models [3]. Recall that the Chow-Liu algorithm computes a tree on random variables $X_{1}, \ldots, X_{n}$ by running a Maximum Spanning Tree algorithm on a weighted clique with nodeset $V=\left\{X_{1}, \ldots, X_{n}\right\}$ and edge weight $w_{i j}$ between every pair of nodes $X_{i}$ and $X_{j}$ that is set equal to an estimate of the mutual information, $I\left(X_{i} ; X_{j}\right)$, between $X_{i}$ and $X_{j}$. (These are precisely Steps 2-4 of Algorithm 1.) When the distribution $\mathrm{P}$ of the random variables is a tree-structured model defined on some tree $T$ wherein the distributions of the endpoints $\left(X_{i}, X_{j}\right)$ of each edge satisfy some non-degeneracy condition, and when the estimation error of each $I\left(X_{i} ; X_{j}\right)$ is small enough, it is known that the computed maximum spanning tree will equal $T$. Moreover, once $T$ known, it is not hard to see-using e.g. the results of [5]-that $O\left(n \ln n / \epsilon^{2}\right)$ samples from $\mathrm{P}$ suffice to define some $\mathrm{Q}$ on this tree such that $d_{\mathrm{TV}}(\mathrm{P}, \mathrm{Q}) \leq \epsilon$.

The above discussion might suggest that a natural learning algorithm for tree-structured models could aim to first identify the tree of some unknown model $\mathrm{P}$, and then identify a model $\mathrm{Q}$ on that tree which is close to P. Unfortunately, it is easy to see that no finite number of samples suffices to identify the true tree even for three variable models. For an obvious example, imagine that the true model $\mathrm{P}$ is a Markov Chain with structure $A \rightarrow B \rightarrow C$. The smaller the mutual informations $I(A, B)$ and $I(B, C)$ are (i.e. the closer to independent these variables become), the more samples are required to distinguish between the true Markov chain structure, and an alternative Markov chain structure, e.g. $B \rightarrow A \rightarrow C$.

Indeed, despite the wide use of the Chow-Liu algorithm and the breadth of follow up work that it has spun for over five decades, we are aware of no work that provides sample-optimal and timeefficient algorithms for learning even tree-structured graphical models, without making assumptions about the model. On the nonefficient learning front, recent work of [7] provides an exponentialtime algorithm which uses an optimal $O\left(\frac{n \ln n}{\epsilon^{2}}\right)$ samples to properly learn an arbitrary tree-structured Ising model, to within total variation distance $\epsilon$. Our main theorem is that Chow-Liu is an efficient algorithm that matches this optimal sample bound.

The main challenge in establishing Theorem 1 lies in dealing with "structural mistakes," that is, the discrepancy between the true tree underlying the distribution that is being learned and the tree output by the Chow-Liu algorithm. Our proof exploits a recent squared Hellinger subadditivity theorem, established in [5] for the purposes of testing the identity of Bayesian networks from samples. It involves an intricate, nesting argument that uses a hierarchical classification of the nodes into groups, induced by a classification of the edges based on some measure of strength, which captures how far from independent their endpoints are. We argue that the edges of the true model may be hard to identify because of them being and/or having in their proximity very strong or very weak edges. Despite the unavoidable mistakes that the algorithm will make in adding edges to the reconstructed tree, we argue that the contribution of these mistakes to the total variation distance between the reconstructed model and the true one can be controlled. Roughly speaking, strong edges partition the nodes into clusters of highly correlated/anti-correlated nodes, and we argue that the algorithm will correctly identify medium strength edges between the correct pairs of clusters, despite possibly assigning them incorrect endpoints. This type of argument, combined with the squared Hellinger subadditivity for bounding the error, is repeated a constant number of times at different scales of edge-strengths. It is worth noting that the partition of the nodes into the hierarchical clustering used in our argument is constructive and based on measures of strength of the edges of the tree $\widehat{T}$ that the Chow-Liu algorithm reconstructs. As a result, a description of the reconstruction guarantees, characterizing the symmetric difference between the true tree and $\widehat{T}$, can be computed explicitly and efficiently. Figures 4 and 6, for respectively symmetric and asymmetric models, illustrate the types of reconstruction guarantees that can be derived from the learned tree. 
A Deterministic Condition for Learning. An interesting feature of our proof is that it identifies a deterministic condition on the sample, which suffices to guarantee that Algorithm 1 will succeed in identifying a tree-structured Ising model $\mathrm{Q}$ that is within $O(\epsilon)$ from $\mathrm{P}$ in total variation distance. This condition, called "Strong 4-Consistency" and presented formally in Definition 23, roughly states that the empirical distribution $\widehat{\mathrm{P}}$ induced by the sample wellapproximates the true distribution $\mathrm{P}$ on all events involving up to 4 variables in the model, and is especially sensitive to small probability events. It can be shown that Strong 4-Consistency holds, with probability at least $1-\gamma$, if we draw $\Omega\left(\frac{n}{\epsilon^{2}} \cdot \ln \frac{n}{\gamma}\right)$ i.i.d. samples from P. Regardless of the provenance of the samples, however, our proof establishes that, if we are handed an empirical distribution $\widehat{\mathrm{P}}$ which satisfies Strong 4-Consistency and use that to run Algorithm 1 from Line 3 onwards, the algorithm will succeed in identifying a treestructured Ising model $\mathrm{Q}$ such that $d_{\mathrm{TV}}(\mathrm{P}, \mathrm{Q}) \leq O(\epsilon)$. In particular, we show the following.

Theorem 2 (Restated as Theorem 24 in Section 4). For $n \in$ $\mathbb{N}, \epsilon, \gamma \in(0,1]$, suppose $\widehat{\mathrm{P}}$ is a distribution over binary variables $\left(X_{1}, \ldots, X_{n}\right)$ satisfying the "Strong 4-Consistency" condition of Definition 23 with respect to distribution $\mathrm{P}$. If we use $\widehat{\mathrm{P}}$ to run Algorithm 1 from Line 3 onwards, the algorithm will succeed in identifying a tree-structured Ising model $\mathrm{Q}$ such that $d_{\mathrm{TV}}(\mathrm{P}, \mathrm{Q}) \leq O(\epsilon)$.

Sample Optimality. We conclude this section by discussing the optimality of the sample requirements in our main theorem. It was shown in [4] (see Theorem 14 and Remark 4 of their paper) that, given sample access to an Ising model $P$, which is known to have a tree structure, one needs $\Omega\left(n / \epsilon^{2}\right)$ samples to distinguish, with success probability at least $2 / 3$, whether $P$ is equal to the uniform distribution over $\{0,1\}^{n}$ versus $\mathrm{P}$ is $\epsilon$-far in total variation distance from the uniform distribution. This immediately yields an $\Omega\left(n / \epsilon^{2}\right)$ lower bound for the problem of properly learning tree-structured Ising models, as the ability to learn implies the ability to test. Using a similar but tighter construction, [11] shows an $\Omega\left(n \ln n / \epsilon^{2}\right)$ sample lower bound for proper learning of tree-structured Ising models with a constant success probability, matching our upper bound.

Roadmap. This is an extended abstract of the work presented in complete form in [6]. The goal of this extended abstract is to convey the main ideas of the proof referring most of the details to [6]. Section 2 provides all the necessary preliminaries for this extended abstract. Section 3 presents a bonus result, that a simple variant of the Chow-Liu algorithm, using absolute correlations rather than mutual informations as weights to build the maximum spanning tree, succeeds in the symmetric case, i.e. when all variables of the tree-structured model that is being learned have marginal distributions that are unbiased. We provide this result because it is simpler to prove and captures most of the main ideas of the general case. We also give it to illustrate the flexibility of our argument, i.e. that it is not specific to the use of mutual information. Section 4 presents our main theorem, i.e. that the Chow-Liu algorithm succeeds in the general case of arbitrary tree-structured models.

\section{PRELIMINARIES}

This section provides the necessary preliminaries for this extended abstract, postponing more details to the complete version [6]. It is well known that the class of binary tree-structured Bayesnets, the class of binary tree-structured Markov Random Fields, and the class of tree-structured Ising models represent the exact same set of distributions. We provide the definition of Bayesian Networks.

Definition 3. A Bayesian network, or Bayesnet, is a joint distribution $\mathrm{P}$ for $X_{1}, \ldots, X_{n}$ with an underlying directed acyclic graph $G$, where each $X_{i}$ takes value in $\mathcal{A}$. To describe $\mathrm{P}$, one specifies conditional probabilities $\mathrm{P}_{i \mid \Pi_{i}}\left(x_{i} \mid x_{\Pi_{i}}\right)$, for all $i \in[n]$, and configurations $x_{i} \in \mathcal{A}$ and $x_{\Pi_{i}} \in \mathcal{A}^{\Pi_{i}}$, where $\Pi_{i}$ represents the set of parents of $i$ in $G$, taken to be $\emptyset$ if $i$ has no parents. In terms of these conditional probabilities, $\mathrm{P}$ assigns to each vector $x \in \mathcal{A}^{n}$ a probability $\mathrm{P}(x)$ satisfying $\mathrm{P}(x)=\prod_{i} \mathrm{P}_{i \mid \Pi_{i}}\left(x_{i} \mid x_{\Pi_{i}}\right)$.

In this paper, we focus on the special case of tree-structured Bayesnets whose underlying DAGs are directed rooted trees with all edges pointing away from the root. We also only consider binary alphabet $\{1,-1\}$ for each variable. We call a Bayesnet symmetric if each variable takes $1,-1$ with probability $\frac{1}{2}$ each.

Given a tree $T$, a subset of nodes is called $T$-connected if they are connected in $T$. Indexes $i_{1}, i_{2} \ldots, i_{r}$ are said to lie on a path in $T$ if $i_{2}$ sits on the path in $T$ between $i_{1}$ and $i_{r}, i_{3}$ sits on the path in $T$ between $i_{2}$ and $i_{r}$, and so on. By path we mean the shortest path (in the tree specified), always.

A tree-structured Bayesnet $\mathrm{P}$ for $X_{1}, \ldots, X_{n}$ can be uniquely determined by specifying its underlying tree $T$, together with its marginal distributions for pairs of variables corresponding to edges of $T$. For a binary symmetric tree-structured Bayesnet $\mathrm{P}$, its marginal distribution for each pair $\left(X_{i}, X_{j}\right)$ (not necessarily an edge of the underlying tree) is determined simply by the probability of the two nodes being equal, namely $\mathrm{P}\left(X_{i}=X_{j}\right)$. For convenience, we reparametrize $\mathrm{P}\left(X_{i}=X_{j}\right)$ using $\alpha_{i j}$ such that $\mathrm{P}\left(X_{i}=X_{j}\right)=\frac{1+\alpha_{i j}}{2}$. We call $\alpha_{i j}$ the $\alpha$-value of the pair $\left(X_{i}, X_{j}\right)$, or just of $(i, j)$, for P. Edges with $\alpha$-values close to 1 or -1 are strong; the two variables are almost always equal or almost always unequal under P. Edges with $\alpha$-values close to 0 are weak; the two variables are nearly independent under $\mathrm{P}$.

The $\alpha$-values satisfy the following nice multiplicativity property: if $i_{1}, i_{2}, \ldots, i_{r}$ lie on a path in $T$, then $\alpha_{i_{1} i_{r}}=\alpha_{i_{1} i_{2}} \ldots \alpha_{i_{r-1} i_{r}}$. For the simple proof see the complete version [6].

We make extensive use of the Hellinger distance, $H$, to help us bound the total variation distance, $d_{\mathrm{TV}}$. The following relationship is known for any distributions $\mathrm{p}^{\prime}$ and $\mathrm{p}^{\prime \prime}: H^{2}\left(\mathrm{p}^{\prime}, \mathrm{p}^{\prime \prime}\right) \leq$ $d_{\mathrm{TV}}\left(\mathrm{p}^{\prime}, \mathrm{p}^{\prime \prime}\right) \leq \sqrt{2} H\left(\mathrm{p}^{\prime}, \mathrm{p}^{\prime \prime}\right)$.

The next corollary, proved in the complete version [6], follows from the subadditivity property of square Hellinger distance [5]. It is important in our analysis for controlling the error when many edges are learned incorrectly.

Corollary 4. Suppose $\mathrm{P}^{\prime}, \mathrm{P}^{\prime \prime}$ are tree-structured Bayesnets on $X_{1}, \ldots, X_{n}$, with underlying trees $T^{\prime}, T^{\prime \prime}$. Suppose $A_{1}, \ldots, A_{\Lambda}$ are nonempty, pairwise disjoint sets whose union is $[n]$, such that

(1) $A_{\lambda}$ is both $T^{\prime}$-connected and $T^{\prime \prime}$-connected, for each $\lambda$;

(2) there is an edge in $T^{\prime}$ between $A_{\lambda}$ and $A_{\mu}$ iff there is an edge in $T^{\prime \prime}$ between $A_{\lambda}$ and $A_{\mu}$, for each pair $\lambda, \mu$.

Let $\mathcal{E}$ be the set of all unordered pairs $(\lambda, \mu)$ such that there is an edge in $T^{\prime}$ and an edge in $T^{\prime \prime}$ straddling $A_{\lambda}$ and $A_{\mu}$. For each $(\lambda, \mu) \in \mathcal{E}$, let $W_{\lambda \mu}$ be the set of endpoints of those two straddling edges, so it has 
cardinality 2, 3, or 4, depending on how many endpoints are shared. Then,

$$
H^{2}\left(\mathrm{P}^{\prime}, \mathrm{P}^{\prime \prime}\right) \leq \sum_{\lambda=1}^{\Lambda} H^{2}\left(\mathrm{P}_{A_{\lambda}}^{\prime}, \mathrm{P}_{A_{\lambda}}^{\prime \prime}\right)+\sum_{(\lambda, \mu) \in \mathcal{E}} H^{2}\left(\mathrm{P}_{W_{\lambda \mu}}^{\prime}, \mathrm{P}_{W_{\lambda \mu}}^{\prime \prime}\right) .
$$

The following notation will be useful later when we reason about the relative positioning of a small subset (3 or 4) of nodes in the underlying tree of the distribution we wish to learn. The subscript at each symbol is a drawing of the underlying line graph of the Markov chain being represented.

DeFINITION 5. Given an arbitrary joint distribution $\mathrm{P}$ for random variables $X_{1}, \ldots, X_{n}$, and $i, j, k \in[n]$, we adopt the following notations:

$\mathrm{P}_{i-j k}$ - the Markov chain for $X_{j}, X_{i}, X_{k}$, in that order, with the marginal for $(i, j)$ equal to $\mathrm{P}_{i j}$, and the marginal for $(i, k)$ equal to $\mathrm{P}_{i k}$;

$\mathrm{P}_{h-i j-k}$ - the Markov chain for $X_{i}, X_{h}, X_{k}, X_{j}$, in that order, with the marginal for $(h, i)$ equal to $\mathrm{P}_{h i}$, the marginal for $(h, k)$ equal to $\mathrm{P}_{h k}$, and the marginal for $(j, k)$ equal to $\mathrm{P}_{j k}$,

The definitions for other examples such as $\mathrm{P}_{i-j-k}, \mathrm{P}_{i \widehat{j-k}}, \mathrm{P}_{h-i \widehat{j-k}}$ are analogous.

\section{THE SYMMETRIC CASE}

As the analysis for general tree-structured Ising models is much more complex, here we first focus on learning symmetric models, wherein every node has a uniform marginal. Even in this case, proving learnability guarantees for the Chow-Liu algorithm is nontrivial, so we focus on a closely related, but slightly easier to analyze algorithm. The analysis of this variation of the Chow-Liu algorithm for proper learning of binary symmetric tree-structured Bayesnets will nevertheless illustrate all the major ideas present in the analysis of the general case. In Section 4, we provide the necessary modifications to our argument to analyze Chow-Liu for general tree-structured Ising models.

Throughout Section 3, we reserve the symbol $\mathrm{P}$ to represent the unknown binary symmetric tree-structured Bayesnet for $X_{1}, \ldots, X_{n}$ that we wish to properly learn. The dimension of $\mathrm{P}$ is $n$. We reserve $T$ to represent the true (but unknown) underlying tree of $\mathrm{P}$, and $\alpha_{i j}$ to represent the true (but unknown) $\alpha$-value of the pair $(i, j)$ under $\mathrm{P}$ (see Section 2 for a discussion of $\alpha$-values). Our proper learning algorithm for the symmetric case is presented as Algorithm 2.

Throughout the section, we will also reserve the symbols $\widehat{\mathrm{P}}, \widehat{\alpha}_{i j}$, $\widehat{T}$, and $\mathrm{Q}$ for the respective meanings they carry in Algorithm 2. We call $\widehat{\alpha}_{i j}$ the $\widehat{\boldsymbol{\alpha}}$-estimate of the pair $(i, j)$, and $\left|\widehat{\alpha}_{i j}\right|$ the $|\widehat{\boldsymbol{\alpha}}|$-estimate of the pair $(i, j)$. Our main result for Algorithm 2 is the following.

Theorem 6. There exists $B>0$ such that for any $\epsilon, \gamma \in(0,1]$, Algorithm 2 outputs a binary symmetric tree-structured Bayesnet $\mathrm{Q}$ such that $d_{\mathrm{TV}}(\mathrm{P}, \mathrm{Q}) \leq \epsilon$, with error probability at most $\gamma$.

In fact, we establish something stronger and perhaps surprising. We show that Algorithm 2 is successful as long as the empirical distribution $\widehat{\mathrm{P}}$ satisfies a deterministic criterion, presented below.

Definition 7 (3-Consistency). For a given run of Algorithm 2, $\widehat{\mathrm{P}}$ is said to satisfy 3-consistency if for every $S \subseteq[n]$ of cardinality

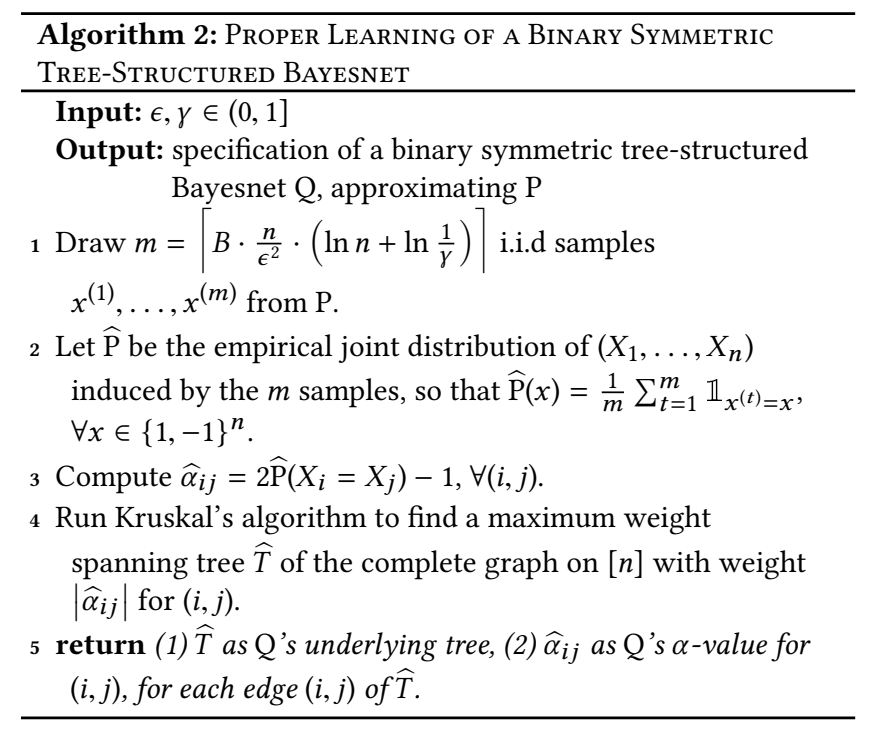

3 and every $W \subseteq\{1,-1\}^{3}$ we have

$\left|\widehat{\mathrm{P}}\left(X_{S} \in W\right)-\mathrm{P}\left(X_{S} \in W\right)\right| \leq \frac{1}{10} \cdot \max \left\{\sqrt{\mathrm{P}\left(X_{S} \in W\right) \cdot \frac{\epsilon^{2}}{n}}, \frac{\epsilon^{2}}{n}\right\}$.

The criterion of Definition 7 specifies that $\widehat{\mathrm{P}}$ well-approximates the true distribution $\mathrm{P}$ for all events involving up to 3 variables. We show that whenever the samples provided to Algorithm 2 induce $\widehat{\mathrm{P}}$ which satisfies 3-consistency, the algorithm succeeds (up to a constant), as per the following theorem.

THEOREM 8. For a run of Algorithm 2, if $\widehat{\mathrm{P}}$ satisfies 3-consistency, then $d_{\mathrm{TV}}(\mathrm{P}, \mathrm{Q}) \leq O(\epsilon)$.

Notice that there are less than $n^{3}$ choices for $S$, and $2^{8}$ choices for $W$ for each $S$. By measure concentration and the union bound, 3-consistency fails with probability at most $\gamma$ if $B$ is chosen large enough. Consequently, showing Theorem 8 implies Theorem 6 (up to a constant). We will focus on proving Theorem 8 .

\subsection{Outline of the Proof}

In this section, we outline our analysis, presenting the intuition guiding our proof as well as the critical techniques that we develop to complete it. The details of our analysis and proofs of the lemmas stated here can be found in the complete version of our paper [6].

Narrowing into a deterministic condition for success. A few words first on the 3-consistency condition (see Definition 7). We'd ideally like to have a simple deterministic condition describing the samples that is satisfied with probability at least $1-\gamma$, and that yields $d_{\mathrm{TV}}(\mathrm{P}, \mathrm{Q}) \leq O(\epsilon)$ if satisfied. Such a deterministic condition serves as an intermediate step between the i.i.d samples and the desired bound in total variation distance between $\mathrm{P}$ and $\mathrm{Q}$. What can we hope for with $m=\left\lceil B \cdot \frac{n}{\epsilon^{2}} \cdot\left(\ln n+\ln \frac{1}{\gamma}\right)\right]$ samples? A simple thing we can hope for is that for each $X_{i}$ the probabilities that it equals 1 or -1 are similar under $\mathrm{P}$ and $\widehat{\mathrm{P}}$. Specifically, by standard Chernoff bounds and the union bound, for any $c>0$ 
we can choose $B$ big enough so that $\left|\widehat{\mathrm{P}}\left(X_{i}=x_{i}\right)-\mathrm{P}\left(X_{i}=x_{i}\right)\right| \leq$ $c \cdot \max \left\{\sqrt{\mathrm{P}\left(X_{i}=x_{i}\right) \cdot \frac{\epsilon^{2}}{n}}, \frac{\epsilon^{2}}{n}\right\}$ for all $i$ and $x_{i} \in\{1,-1\}$, with probability at least $1-\gamma$. In fact, for any fixed constant $K$, by choosing a big enough $B$ we can get similar guarantees simultaneously for all events of the form " $X_{S} \in W$ " for all subsets $S$ of $K$ indexes and all $W \subseteq\{1,-1\}^{K}$, with probability at least $1-\gamma$. Our surprising finding is that taking $K=3$ (and $c=\frac{1}{10}$ ) is sufficient to guarantee the success of the algorithm.

From now on we assume that $\widehat{P}$ satisfies 3-consistency. It suffices to show that this alone implies $d_{\mathrm{TV}}(\mathrm{P}, \mathrm{Q}) \leq O(\epsilon)$, for the output $Q$ of the algorithm (that is, it suffices to prove Theorem 8). It is worth emphasizing that our argument from this point on is completely deterministic. In fact, if $\widehat{\mathrm{P}}$ were obtained in some way other than being the empirical joint distribution of i.i.d samples (e.g., from dependent samples or some oracle), as long as it satisfies 3-consistency Algorithm 2 would still output Q such that $d_{\mathrm{TV}}(\mathrm{P}, \mathrm{Q}) \leq O(\epsilon)$.

Long hybrid arguments vs squared Hellinger subadditivity. We first illustrate how $H$ (Hellinger distance) and the subadditivity of $H^{2}$ (squared Hellinger) play an important role in the argument even though our final goal is to provide a bound in $d_{\mathrm{TV}}$ (total variation distance). Suppose, for the sake of this illustration, that we happened to learn the structure of the tree correctly, that is $\widehat{T}=T$. In this case, $\mathrm{P}$ and $\mathrm{Q}$ would only differ in their $\alpha$-values on the edges of the true $T$ $\left(\alpha_{i j}\right.$ for $\mathrm{P}$ and $\widehat{\alpha}_{i j}$ for $\left.\mathrm{Q}\right)$. How could we bound $d_{\mathrm{TV}}(\mathrm{P}, \mathrm{Q})$ ? One way is to use a hybrid argument modifying $\mathrm{P}$ to $\mathrm{Q}$ by changing the $\alpha$-value of one edge of $T$ at a time from $\alpha_{i j}$ to $\widehat{\alpha}_{i j}$, resulting in a sequence of intermediate distributions all with the same underlying tree $T$ and with increasingly more edges of $T$ having $\alpha$-value $\widehat{\alpha}_{i j}$ instead of $\alpha_{i j}$. It easily follows from 3-consistency that the difference between $\alpha_{i j}$ and $\widehat{\alpha}_{i j}$ is $O\left(\max \left\{\sqrt{\left(1-\left|\alpha_{i j}\right|\right) \cdot \frac{\epsilon^{2}}{n}}, \frac{\epsilon^{2}}{n}\right\}\right)$, and this bounds the cost in $d_{\mathrm{TV}}$ when we change the $\alpha$-value of an edge from $\alpha_{i j}$ to $\widehat{\alpha}_{i j}$. In general, this cost is upper bounded by $O\left(\frac{\epsilon}{\sqrt{n}}\right)$, so for $n-1$ changes of $\alpha$-value the triangle inequality gives an overall $d_{\text {TV }}$ bound between $P$ and $Q$ of $O(\sqrt{n} \epsilon)$, which is not good enough

$H$ suffers from the same problem. It can be shown that the cost in $H$ when we change the $\alpha$-value of an edge from $\alpha_{i j}$ to $\widehat{\alpha}_{i j}$ is $O\left(\frac{\epsilon}{\sqrt{n}}\right)$ (for a proof see the complete version [6]), which is also not enough to make the hybrid argument work. But, here is an important insight. The $H^{2}$ subadditivity property (see Corollary 4 ) applied to our case implies that $H^{2}(\mathrm{P}, \mathrm{Q})$ is bounded by the sum of $H^{2}\left(\mathrm{P}_{i j}, \mathrm{Q}_{i j}\right)$ for all edges $(i, j)$ of $T$. Note that $H^{2}$ is not a distance metric, so the subadditivity is not the result of some hybrid argument changing the $\alpha$-values one edge at a time. Rather, we are changing the $\alpha$ values for all $n-1$ edges of $T$ in one round. Since we pay $O\left(\frac{\epsilon^{2}}{n}\right)$ in $H^{2}$ per edge, in total we pay $O\left(\epsilon^{2}\right)$ in squared Hellinger, yielding $d_{\mathrm{TV}}(\mathrm{P}, \mathrm{Q}) \leq \sqrt{2} H(\mathrm{P}, \mathrm{Q}) \leq O(\epsilon)$, as desired.

Causes of structural uncertainty. Of course, we can't expect $\widehat{T}=T$. It is possible that $\widehat{T}$ may be very different from $T$. If a subset of nodes are linked together in $T$ by edges with $\alpha$-values 1 , then those nodes will always equal each other in the sample, and there is no way Algorithm 2 can tell what the true edges are. Algorithm 2 will be similarly clueless if the linking edges all have $\alpha$-values 1 or -1 , or if they all have $\alpha$-values 0 (in which case the variables will be mutually independent). Intuitively, we should expect Algorithm 2 to also have difficulty picking out the true edges if the $\alpha$-values are close enough to 1 or -1 , or close enough to 0 . When different strength scales interact the algorithm may get a "partial clue" of the structure. For example, suppose $n=3$ and $X_{1}, X_{2}, X_{3}$ form a Markov chain, in that order, with $\alpha_{12}=\frac{1}{2}$ and $\alpha_{23}$ close to 1 . Then Algorithm 2 should be able to pick out edge $(2,3)$, but might have trouble distinguishing between $(1,2)$ and $(1,3)$ (in other words, $\left|\widehat{\alpha}_{23}\right|$ should be the clear winner but the order between $\left|\widehat{\alpha}_{12}\right|$ and $\left|\widehat{\alpha}_{13}\right|$ might be reversed). The case $\alpha_{12}$ close to 0 and $\alpha_{23}=\frac{1}{2}$ is similar.

As $n$ gets larger the picture gets complicated very quickly, and it seems very hard to reason about the effect of interactions among different edges and the order relationships among different $\left|\widehat{\alpha}_{i j}\right|$ 's. The central difficulty is controlling the error when $\widehat{T}$ and $T$ differ in many edges. The main difficulty is to bound the error when switching the edges from those of $T$ to those of $\widehat{T}$. Once that is done, further switching the $\alpha$-values of the edges of $\widehat{T}$ from $\alpha_{i j}$ to $\widehat{\alpha}_{i j}$ (with the underlying tree fixed to be $\widehat{T}$ ) can be dealt with relatively easily as illustrated above. More specifically, if we define the hybrid distribution $\mathrm{P}^{(4)}$ (the reason for calling it $\mathrm{P}^{(4)}$ will be clear later), with underlying tree $\widehat{T}$ and $\alpha$-value equal to $\alpha_{i j}$ for every edge $(i, j)$ in $\widehat{T}$, it follows from our earlier $H^{2}$-subadditivity argument that $d_{\mathrm{TV}}\left(\mathrm{P}^{(4)}, \mathrm{Q}\right) \leq O(\epsilon)$. So our focus from now on is bounding $d_{\mathrm{TV}}\left(\mathrm{P}, \mathrm{P}^{(4)}\right)$.

Some modest understanding. Let's start simple and consider only three nodes $i, j, k$ that lie on a path in $T$ ( $n$ can be arbitrary, and $i, j, k$ don't have to be adjacent to each other). The multiplicativity of $\alpha$-values along a path implies that $\alpha_{i k}=\alpha_{i j} \alpha_{j k}$ is no greater in absolute value than either $\alpha_{i j}$ or $\alpha_{j k}$. Algorithm 2 may "reverse the order" between $(i, j)$ and $(i, k)$ if $\left|\widehat{\alpha}_{i j}\right| \leq\left|\widehat{\alpha}_{i k}\right|$. The following lemma characterizes when this can happen (assuming 3-consistency):

Lemma 9. If $i, j, k$ lie on a path in $T$, and $\left|\widehat{\alpha}_{i j}\right| \leq\left|\widehat{\alpha}_{i k}\right|$, then $\alpha_{i j}^{2}\left(1-\left|\alpha_{j k}\right|\right) \leq \frac{\epsilon^{2}}{n}$.

The next lemma bounds the $H^{2}$ distance between $\mathrm{P}_{i j k}$ and $\mathrm{P}_{i \widehat{j-k}}$ (see Definition 5 for the definitions of symbols such as $\mathrm{P}_{i \widehat{j-k}}$ ). It is the error in $H^{2}$ when we switch from $(i, j)$ to $(i, k)$. See Figure 1 for depictions of those two distributions.

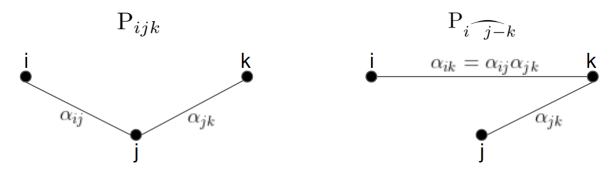

Figure 1: Depictions of $\mathrm{P}_{i j k}$ and $\mathrm{P}_{i \widehat{j-k}}$ in terms of their underlying trees and their $\alpha$-values for the edges of the respective underlying trees.

LemmA 10. If $i, j, k$ lie on a path in $T$, then $H^{2}\left(\mathrm{P}_{i j k}, \mathrm{P}_{i} \widehat{j-k}\right) \leq$ $2 \alpha_{i j}^{2}\left(1-\left|\alpha_{j k}\right|\right)$.

The next lemma follows directly from Lemmas 9 and 10. It says that if Algorithm 2 makes a structural mistake and "reverses the 
order" between $(i, j)$ and $(i, k)$, then the resulting error in $H^{2}$ between the true structure and the wrong structure must be small (assuming 3-consistency).

Lemma 11. If $i, j, k$ lie on a path in $T$, and $\left|\widehat{\alpha}_{i j}\right| \leq\left|\widehat{\alpha}_{i k}\right|$, then $H^{2}\left(\mathrm{P}_{i j k}, \mathrm{P}_{i \widehat{j-k}}\right) \leq 2 \frac{\epsilon^{2}}{n}$.

We also have the following four-node version of Lemma 11 (see Figure 2 for depictions of the two distributions involved):

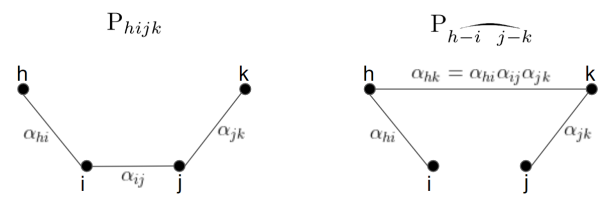

Figure 2: Depictions of $\mathrm{P}_{h i j k}$ and $\mathrm{P}_{h-i j-k}$ in terms of their underlying trees and their $\alpha$-values for the edges of the respective underlying trees.

LEMMA 12. If $h, i, j, k$ lie on a path in $T$, and $\left|\widehat{\alpha}_{i j}\right| \leq\left|\widehat{\alpha}_{h k}\right|$, then $H^{2}\left(\mathrm{P}_{h i j k}, \mathrm{P}_{h-i j-k}\right) \leq 8 \frac{\epsilon^{2}}{n}$.

The challenges of going bigger. It is not hard to see using Lemmas 11 and 12 that if Algorithm 2 picks a single wrong edge, then $H\left(\mathrm{P}, \mathrm{P}^{(4)}\right) \leq O\left(\frac{\epsilon}{\sqrt{n}}\right)$. So what if the algorithm picks multiple wrong edges? An obvious idea is to use the triangle inequality (satisfied by $H$ ) and create many intermediate distributions between $\mathrm{P}$ and $\mathrm{P}^{(4)}$, going from $T$ to $\widehat{T}$ replacing one edge at a time (possibly through the use of some intermediate edges that belong to neither $T$ nor $\widehat{T})$, assigning to each edge $(i, j)$ of the underlying tree of each intermediate distribution $\alpha$-value $\alpha_{i j}$, and using Lemmas 11 and 12 to bound the error in $H$ after each edge replacement. Unfortunately, this does not work, not only because the triangle inequality would lead to a bound that is potentially $\sqrt{n}$ times too big, but also because there are examples of $T$ and $\widehat{T}$ for which there does not even exist a sequence of replacements that would allow us to properly apply Lemmas 11 or 12 to bound the error in $H$ after every replacement. ${ }^{1}$ See Figure 3 for such a pair of $T$ and $\widehat{T}$.

To salvage the situation we choose to use Corollary 4 (squared Hellinger subadditivity) which allows us to replace many edges in one round, as long as they are "compatible," as per the statement of that corollary. If we allow $r$ general rounds of edge replacements, then we may need to replace $\Theta\left(\frac{n}{r}\right)$ edges per round. Assume that either Lemma 11 or 12 is applicable for each individual edge replacement, then we can get an error bound of $O\left(\sqrt{\frac{n}{r} \cdot \frac{\epsilon^{2}}{n}}\right)=O\left(\frac{\epsilon}{\sqrt{r}}\right)$ in $H$ per round, and so $O(\sqrt{r} \epsilon)$ in $H$ in total. This suggests aiming for a constant number of rounds. It is not clear at all how this might be achieved. For that, we introduce another crucial structural

\footnotetext{
${ }^{1}$ To get a sense of the potential issue, say for one of the steps we replace $(i, j)$ with $(h, k)$. Say $h$ is closer to $i$ than to $j$ with respect to the intermediate tree right before the replacement. Then, in order for us to be able to apply Lemma 12, we need for example that the $\alpha$-value of $(h, i)$ for the intermediate distribution right before the replacement to be equal to $\alpha_{h i}$, which is the $\alpha$-value of $(h, i)$ for P. This may not hold due to all the edge replacements that occurred up to this step, which may have created extra nodes on the path in the intermediate tree between $h$ and $i$ that are not on the path in $T$ between $h$ and $i$.
}

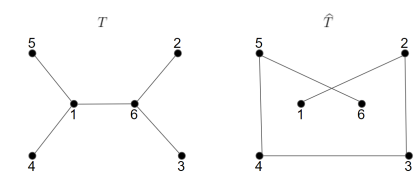

Figure 3: A pair of $T$ and $\widehat{T}$ for which there is no way to go from $T$ to $\widehat{T}$ by replacing one edge at a time in a way that allows us to properly apply Lemmas 11 and 12 to bound the error in $H$ after every replacement.

ingredient-layering-which allows us to be more precise about the manner in which $\widehat{T}$ may differ from $T$.

Building a hierarchy. We classify the edges of $\widehat{T}$ based on their $|\widehat{\alpha}|$-estimates. This induces a hierarchical classification of nodes into groups, which in turn induces a classification of the edges of $T{ }^{2}$ We emphasize that this entire layering is merely a thought experiment to facilitate the proof.

At the top level of our layering, we classify an edge $(i, j)$ of $\widehat{T}$ as a $\widehat{T}$-road if $\left|\widehat{\alpha}_{i j}\right| \geq 1-10 \frac{\epsilon^{2}}{n}$. The $\widehat{T}$-roads cluster the $n$ nodes into connected components, each of which is called a city. An edge $(i, j)$ of $T$ with $i, j$ belonging to the same city is classified as a $T$-road. We can prove the following structural results.

LEMmA 13. If $(i, j)$ is a T-road or a $\widehat{T}$-road, then $\left|\alpha_{i j}\right| \geq 1-11 \frac{\epsilon^{2}}{n}$.

LEMMA 14. If $i, j$ belong to different cities, then $\left|\alpha_{i j}\right|<1-9 \frac{\epsilon^{2}}{n}$.

LEMMA 15. Every city is T-connected.

Lemma 15 says that each city is not only spanned by the $\widehat{T}$-roads in it (this is by definition), but also spanned by the $T$-roads in it. Intuitively, the $T$-roads and $\widehat{T}$-roads are so strong that within each city it doesn't make much difference (in terms of $H^{2}$ ) if the nodes were connected by $T$-roads or by $\widehat{T}$-roads. Later, we will replace the set of all $T$-roads with the set of all $\widehat{T}$-roads in a single round of edge replacement in the process of going from $T$ to $\widehat{T}$.

For the next level of our layering, we classify an edge $(i, j)$ of $\widehat{T}$ as a $\widehat{T}$-highway if $\frac{1}{2} \leq\left|\widehat{\alpha}_{i j}\right|<1-10 \frac{\epsilon^{2}}{n}$. The $\widehat{T}$-highways cluster the cities into bigger connected components, each of which is called a country. An edge $(i, j)$ of $T$ with $i, j$ belonging to different cities in the same country is classified as a $\boldsymbol{T}$-highway. We can prove the following structural results.

LEMMA 16. There is a $\widehat{T}$-highway between a pair of cities if and only if there is a T-highway between the same pair of cities.

Proof. (sketch of the "only if" direction). Suppose for the sake of contradiction that $(i, k)$ is a $\widehat{T}$-highway between cities $C$ and $D$, and that there is no edge of $T$ between $C$ and $D$ (any such edge would in fact be classified as a $T$-highway because $C$ and $D$ are clearly different cities in the same country). Then, the path in $T$

\footnotetext{
${ }^{2}$ We could have let $T$ dictate the layering, starting by classifying the edges of $T$ instead. The proof would be somewhat more complicated if we went this way. We could also have chosen to base the classification on $|\alpha|$-values rather than $|\widehat{\alpha}|$-estimates. We chose $|\widehat{\alpha}|$-estimates because those are known after running Algorithm 2 (just like $\widehat{T}$ is). This will enable us to actually tell which edge of $\widehat{T}$ is classified as what, and visualize the partitioning of nodes into groups and therefore various reconstruction guarantees obtained by the algorithm.
} 
between $i$ and $k$ must contain some $j$ that belongs to neither $C$ nor $D$. Since $\left|\widehat{\alpha}_{i k}\right| \geq \frac{1}{2}$, by 3-consistency $\left|\alpha_{i k}\right| \geq \frac{1}{2}-\frac{1}{5} \frac{\epsilon}{\sqrt{n}}$, and by the multiplicativity of $\alpha$-values $\left|\alpha_{i j}\right| \geq \frac{1}{2}-\frac{1}{5} \frac{\epsilon}{\sqrt{n}}$. Since $j, k$ belong to different cities, by Lemma $14\left|\alpha_{j k}\right| \leq 1-9 \frac{\epsilon^{2}}{n}$. Since $\left(\frac{1}{2}-\frac{1}{5} \frac{\epsilon}{\sqrt{n}}\right)^{2} \cdot 9 \frac{\epsilon^{2}}{n}>\frac{\epsilon^{2}}{n}$, it follows from (the contrapositive of) Lemma 11 that $\left|\widehat{\alpha}_{i j}\right|>\left|\widehat{\alpha}_{i k}\right|$. Symmetrically we also have $\left|\widehat{\alpha}_{j k}\right|>$ $\left|\widehat{\alpha}_{i k}\right|$. Together they imply that $(i, k)$ could not possibly be picked to be an edge of $\widehat{T}$ by the maximum spanning tree algorithm.

\section{LEMMA 17. Every country is T-connected.}

LEMMA 18. If $i, j$ belong to different countries, then $\left|\alpha_{i j}\right|<\frac{1}{2}+$ $\frac{1}{5} \frac{\epsilon}{\sqrt{n}}$.

The lower threshold $\frac{1}{2}$ in the criterion for $\widehat{T}$-highways has been chosen in view of Lemmas 9 and 14 so that Lemma 16 will work. We want to roughly aim for (lower threshold for $\widehat{T}$-highways) ${ }^{2}$. $(1-$ lower threshold for $\widehat{T}$-roads $)>\frac{\epsilon^{2}}{n}$. Please refer to the inequality $\left(\frac{1}{2}-\frac{1}{5} \frac{\epsilon}{\sqrt{n}}\right)^{2} \cdot 9 \frac{\epsilon^{2}}{n}>\frac{\epsilon^{2}}{n}$ in the proof sketch above for a precise version that takes estimation errors into account. Lemma 16 says that the $T$-highways and $\widehat{T}$-highways can be matched into parallel pairs, where each parallel pair consists of a $T$-highway and a $\widehat{T}$-highway going between the same pair of cities (in the same country). Later, we will replace the set of all $T$-highways with the set of all $\widehat{T}$-highways in a single round of edge replacement in the process of going from $T$ to $\widehat{T}$.

For the next level of our layering, we classify an edge $(i, j)$ of $\widehat{T}$ as a $\widehat{T}$-railway if $2 \frac{\epsilon}{\sqrt{n}} \leq\left|\widehat{\alpha}_{i j}\right|<\frac{1}{2}$. The $\widehat{T}$-railways cluster the countries into bigger connected components, each of which is called a continent. An edge $(i, j)$ of $T$ with $i, j$ belonging to different countries in the same continent is classified as a $T$-railway. We can prove the following structural results.

LEMMA 19. There is a $\widehat{T}$-railway between a pair of countries if and only if there is a T-railway between the same pair of countries.

\section{Lemma 20. Every continent is T-connected.}

LEMma 21. If $i, j$ belong to different continents, then $\left|\alpha_{i j}\right|<\frac{11}{5} \frac{\epsilon}{\sqrt{n}}$.

The proof of Lemma 19 is similar to that of Lemma 16. The lower threshold $2 \frac{\epsilon}{\sqrt{n}}$ in the criterion for $\widehat{T}$-railways has been chosen in view of Lemma 9 and Lemma 18 so that Lemma 19 will work. We want to roughly aim for (lower threshold for $\widehat{T}$-railways) $)^{2}$. $(1-$ lower threshold for $\widehat{T}$-highways $)>\frac{\epsilon^{2}}{n}$. Lemma 19 says that the $T$-railways and $\widehat{T}$-railways can be matched into parallel pairs, where each parallel pair consists of a $T$-railway and a $\widehat{T}$-railway going between the same pair of countries (in the same continent). Later, we will replace the set of all $T$-railways with the set of all $\widehat{T}$-railways in a single round of edge replacement in the process of going from $T$ to $\widehat{T}$.

At the bottom layer, we classify the remaining edges of $\widehat{T}$ as $\widehat{T}$-airways, and the remaining edges of $T$ as $\boldsymbol{T}$-airways. Those are edges that go between different continents. Intuitively, the $T$ airways and $\widehat{T}$-airways are so weak (with $\alpha$-value $O\left(\frac{\epsilon}{\sqrt{n}}\right)$ ) that it doesn't make much difference (in terms of $H^{2}$ ) if the continents were linked together with each other by $T$-airways or by $\widehat{T}$-airways. In fact, those edges can be cut (i.e. change the $\alpha$-value to 0 ) without incurring too much error. Later, we will replace the set of all $T$ airways with the set of all $\widehat{T}$-airways in a single round of edge replacement in the process of going from $T$ to $\widehat{T}$.

Summary of layering. To summarize, the edges of $\widehat{T}$ are classified into $\widehat{T}$-roads, $\widehat{T}$-highways, $\widehat{T}$-railways, and $\widehat{T}$-airways based on their $|\widehat{\alpha}|$-estimates. The $n$ nodes are clustered into cities by the $\widehat{T}$-roads, which are further clustered into countries by the $\widehat{T}$-highways, which are further clustered into continents by the $\widehat{T}$-railways, which are finally linked into one component by the $\widehat{T}$-airways. This hierarchical classification of nodes into groups then induces a classification of the edges of $T$ : an edge of $T$ is classified as a $T$-road if its two end-nodes belong to the same city, a $T$-highway if its two end-nodes belong to different cities in the same country, a $T$-railway if its two end-nodes belong to different countries in the same continent, or a $T$-airway if its two end-nodes belong to different continents.

Note that each city, country, or continent is $\widehat{T}$-connected by definition. We can prove that every city, country, or continent is also $T$-connected. Furthermore, we can prove that the $T$-highways and $\widehat{T}$-highways can be matched into parallel pairs, that is, there is a $\widehat{T}$-highway between two cities if and only if there is a $T$-highway between them. The same goes for railways: there is a $\widehat{T}$-railway between two countries if and only if there is a $T$-railway between them. One consequence of everything we stated so far is that the exact same clusters (i.e. cities, countries, or continents) at each level of the hierarchy would have been achieved if we were to cluster the $n$ nodes first by $T$-roads, then $T$-highways, then $T$-railways, and finally $T$-airways.

For a diagrammatic illustration of our structural results see Figure 4. The solid connections represent edges of $T$, and the dashed connections represent edges of $\widehat{T}$. The thickness of the line increases as we go down in the hierarchy, with the roads being the thinnest and the airways being the thickest. The circles represent the cities, the rectangular boxes represent the countries, and the saw-toothed regions represent the continents. Note that the $T$-highways and $\widehat{T}$-highways can be matched into parallel pairs, each going between the same pair of cities (in the same country). An example is $(1,2)$ and $(3,4)$. Similarly, the $T$-railways and $\widehat{T}$-railways can be matched into parallel pairs, each going between the same pair of countries (in the same continent). An example is $(5,6)$ and $(7,8)$. This parallelism needs not hold for roads and airways. For example, there is no $\widehat{T}$-airway that goes between the same pair of continents as the $T$-airway $(9,10)$ does, and there is no $T$-airway that goes between the same pair of continents as the $\widehat{T}$-airway $(11,12)$ does.

Putting everything together. We are now ready to bound $d_{\mathrm{TV}}(\mathrm{P}, \mathrm{Q})$. Recall that $\mathrm{P}^{(4)}$ was defined as the distribution with underlying tree $\widehat{T}$ and $\alpha$-value equal to $\alpha_{i j}$ for every edge $(i, j)$ in $\widehat{T}$. We already argued that $d_{\mathrm{TV}}\left(\mathrm{P}^{(4)}, \mathrm{Q}\right) \leq O(\epsilon)$, so it suffices to bound $H\left(\mathrm{P}, \mathrm{P}^{(4)}\right)$ (remember $d_{\text {TV }}$ is upper bounded by $\sqrt{2}$ times $H$ ). There will be four rounds of edge replacements to go from $T$ to $\widehat{T}$. Starting with $T$, first we replace the set of all $T$-airways by the set of all $\widehat{T}$-airways, then replace the set of all $T$-railways by the set of all $\widehat{T}$-railways, 


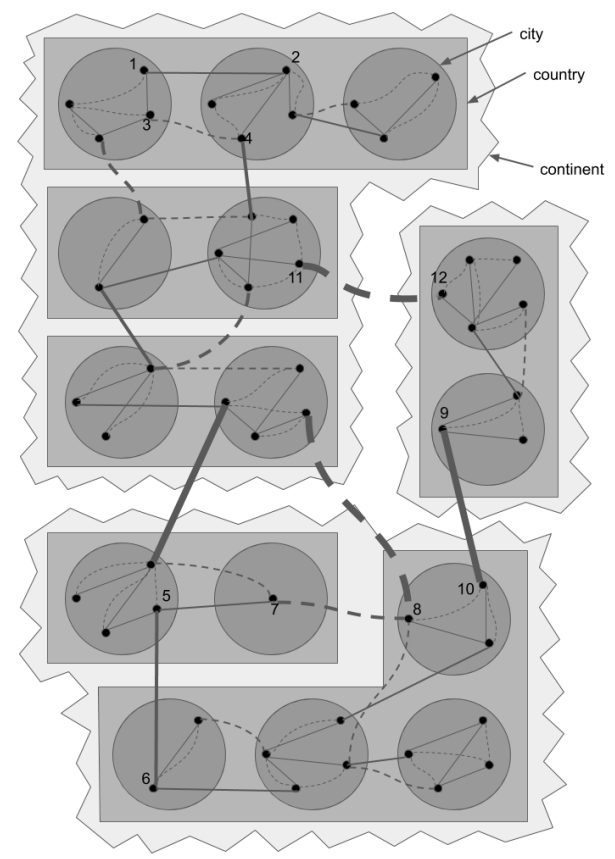

Figure 4: A diagrammatic illustration of our hierarchy. For a description of the figure see the last paragraph of "Summary of layering" in Section 3.1.

then replace the set of all $T$-highways by the set of all $\widehat{T}$-highways, and finally replace the set of all $T$-roads by the set of all $\widehat{T}$-roads (as will soon be seen, it is crucial that we do those edge replacements in a bottom-up fashion, starting with the airways and ending with the roads). This creates three intermediate distributions $\mathrm{P}^{(1)}, \mathrm{P}^{(2)}, \mathrm{P}^{(3)}$ between $\mathrm{P}$ and $\mathrm{P}^{(4)}$. The underlying trees of $\mathrm{P}^{(1)}, \mathrm{P}^{(2)}, \mathrm{P}^{(3)}$ are various hybrids between $T$ and $\widehat{T}$ (for example, the underlying tree of $\mathrm{P}^{(2)}$ consists of the $T$-roads, the $T$-highways, the $\widehat{T}$-railways, and the $\widehat{T}$-airways). For each of these distributions the $\alpha$-value for each edge $(i, j)$ of its underlying tree is $\alpha_{i j}$.

To bound $H\left(\mathrm{P}, \mathrm{P}^{(1)}\right)$, we perform one sub-step in which we "cut" all the $T$-airways (i.e. change their $\alpha$-values to 0 ) so that the continents become mutually independent, and another step in which we "install" all the $\widehat{T}$-airways. For each of those two sub-steps, we apply Corollary 4 with $A_{1}, \ldots, A_{\Lambda}$ being the continents. The potentially nonzero terms on the right hand of (1) in Corollary 4 are those in the second summand, with the $W_{\lambda \mu}$ 's corresponding to the $T$-airways for the first sub-step, and to the $\widehat{T}$-airways for the second sub-step. By Lemma 21, every $T$-airway or $\widehat{T}$-airway has $|\alpha|$-value $O\left(\frac{\epsilon}{\sqrt{n}}\right)$. It can be shown (see [6]) that such an edge is $O\left(\frac{\epsilon^{2}}{n}\right)$ away in $H^{2}$ from an edge with $\alpha$-value 0 . Since there are less than $n T$-airways and $\widehat{T}$-airways, Corollary 4 implies a loss of $O(\epsilon)$ in $H$ in each of the two sub-steps.

To bound $H\left(\mathrm{P}^{(1)}, \mathrm{P}^{(2)}\right)$, we apply Corollary 4 with $A_{1}, \ldots, A_{\Lambda}$ being the countries. The potentially nonzero terms on the right hand side of (1) in Corollary 4 are those in the second summand, each of which corresponds to switching between a parallel pair of
$T$-railway and $\widehat{T}$-railway that go between the same pair of countries ( $W_{\lambda \mu}$ is the set of endpoints of those two railways). By Lemma 12 (or Lemma 11 if the parallel pair of railways share an end-node), each such switch costs us $O\left(\frac{\epsilon^{2}}{n}\right)$ in $H^{2}$. Note that those lemmas are indeed applicable because each country is both $T$-connected and $\widehat{T}$-connected, and that the edges within each country haven't been replaced yet and are still edges of the true tree $T$; here we see that it is crucial to replace the edges from the bottom layer to the top layer). So overall we lose $O(\epsilon)$ in $H$ in this round.

The bounding of $H\left(\mathrm{P}^{(2)}, \mathrm{P}^{(3)}\right)$ is similar; we apply Corollary 4 with $A_{1}, \ldots, A_{\Lambda}$ being the cities.

Finally, to bound $H\left(\mathrm{P}^{(3)}, \mathrm{P}^{(4)}\right)$, we first "tighten" all the $T$-roads (i.e. turn their $\alpha$-values to 1 or -1 depending on which is closer). At this point we can equivalently think of each city as being held together by the $\widehat{T}$-roads in it with appropriately chosen 1 or -1 as $\alpha$-values. We then "relax" all the $\widehat{T}$-roads back to their original $\alpha$ values. For each of those two sub-steps we apply Corollary 4 with $A_{1}, \ldots, A_{\Lambda}$ being the individual nodes. The potentially nonzero terms on the right hand of (1) in Corollary 4 are those in the second summand, with the $W_{\lambda \mu}$ 's corresponding to the $T$-roads for the first sub-step, and to the $\widehat{T}$-roads for the second sub-step. Every $T$-road or $\widehat{T}$-road has $|\alpha|$-value that is $1-O\left(\frac{\epsilon^{2}}{n}\right)$. It can be shown (see [6]) that "tightening" or "relaxing" such an edge costs $O\left(\frac{\epsilon^{2}}{n}\right)$ in $H^{2}$. Since there are less than $n T$-roads and $\widehat{T}$-roads, Corollary 4 implies a loss of $O(\epsilon)$ in $H$ in each of the two sub-steps.

\section{THE GENERAL CASE}

In this section, we discuss our main result for proper learning general binary-alphabet tree-structured Bayesnets. In particular, we show that this is achieved by the Chow-Liu algorithm using the plug-in estimator for estimating all required mutual information quantities (or equivalently, by running the Chow-Liu algorithm on the empirical joint distribution induced by the samples). Our upper bound from Theorem 1 is restated as Theorem 22 below.

Throughout Section 4, we reserve the symbol P to represent the unknown binary tree-structured Bayesnet on $X_{1}, \ldots, X_{n}$ that we wish to properly learn. The dimension of $\mathrm{P}$ is $n$. We reserve $T$ to represent the true (but unknown) underlying tree of $P$.

The Chow-Liu Algorithm, using the plug-in estimator for estimating mutual information, is presented in the introduction as Algorithm 1. Throughout this section, we will also reserve the symbols $\widehat{\mathrm{P}}, \widehat{T}$, and $\mathrm{Q}$ for the respective meanings they carry in Algorithm 1. Our main result for Algorithm 1 is the following:

Theorem 22. There exists $B>0$ such that for any $\epsilon, \gamma \in(0,1]$, Algorithm 1 specifies a binary tree-structured Bayesnet $\mathrm{Q}$ such that $d_{\mathrm{TV}}(\mathrm{P}, \mathrm{Q}) \leq \epsilon$, with error probability at most $\gamma$.

In fact, we establish something stronger and perhaps surprising. We show that Algorithm 1 is successful as long as the empirical distribution $\widehat{\mathrm{P}}$ satisfies a deterministic criterion, presented below.

Definition 23 (4-Consistency, Strong 4-Consistency). For a given run of Algorithm 1, $\widehat{\mathrm{P}}$ is said to satisfy 4-consistency if 


$$
\begin{aligned}
& \text { for every } S \subseteq[n] \text { of cardinality } 4 \text { and every } W \subseteq\{1,-1\}^{4} \text { we have } \\
& \left|\widehat{\mathrm{P}}\left(X_{S} \in W\right)-\mathrm{P}\left(X_{S} \in W\right)\right| \leq \frac{1}{10^{20}} \cdot \max \left\{\sqrt{\mathrm{P}\left(X_{S} \in W\right) \cdot \frac{\epsilon^{2}}{n}}, \frac{\epsilon^{2}}{n}\right\}
\end{aligned}
$$

It is said to satisfy strong 4-consistency if furthermore whenever $\mathrm{P}\left(X_{S} \in W\right)<\frac{\epsilon^{2}}{n}$ we also have

$$
\widehat{\mathrm{P}}\left(X_{S} \in W\right) \cdot \ln \frac{\frac{\epsilon^{2}}{n}}{\mathrm{P}\left(X_{S} \in W\right)} \leq \frac{\epsilon^{2}}{n} .
$$

The criterion (3) of Definition 23 specifies that $\widehat{\mathrm{P}}$ well-approximates the true distribution $\mathrm{P}$ for all events involving up to 4 variables, and the criterion (4) is a strengthening of (3) for events (involving up to 4 variables) that have small probabilities under $\mathrm{P}$. We show that whenever the samples provided to Algorithm 1 induce $\widehat{P}$ which satisfies strong 4-consistency, the algorithm succeeds (up to a constant), as per the following theorem.

TheOREM 24. For a run of Algorithm 1, if $\widehat{\mathrm{P}}$ satisfies strong 4consistency, then $d_{\mathrm{TV}}(\mathrm{P}, \mathrm{Q}) \leq O(\epsilon)$.

Notice that there are less than $n^{4}$ choices for $S$, and $2^{16}$ choices for $W$ for each $S$. Using concentration of measure and the union bound, it can be shown (see [6]) that strong 4-consistency fails with probability at most $\gamma$ if $B$ is chosen large enough. Consequently, showing Theorem 24 implies Theorem 22 (up to a constant). We will focus on proving Theorem 24 .

\subsection{Outline of the Proof}

In this section, we outline our proof for the general case. The proof that the Chow-Liu algorithm (Algorithm 1) succeeds in properly learning arbitrary binary tree-structured Bayesnets employs the same basic ideas - namely, the squared Hellinger subadditivity and layering - as our proof that the Chow-Liu variant (Algorithm 2) succeeds in the symmetric case, given in Section 3. However, the general case involves a few new challenges and the argument is much more complicated. Our proof sketch in this section will be less detailed than the proof sketch in Section 3.1 for the symmetric case, and we will focus mainly on the new challenges encountered in the general case and the necessary modifications required, as compared to the symmetric case. The proofs of all lemmas in this section and the complete details of our proof are given in the complete version of our paper [6].

The deterministic condition for the success of Chow-Liu. Recall that when analyzing Algorithm 2 in the symmetric case we were able to identify a deterministic condition on the relationship between $\mathrm{P}$ and $\widehat{\mathrm{P}}$, called 3-consistency, that holds with probability at least $1-\gamma$ for a run of Algorithm 2 (for a big enough constant $B$ ) and that can be shown to imply the desired bound $d_{\mathrm{TV}}(P, Q) \leq O(\epsilon)$. Roughly speaking, 3-consistency says that $\mathrm{P}$ and $\widehat{\mathrm{P}}$ assign similar probabilities to all events involving up to three variables. For the general case we need something a bit stronger. First, we need precision guarantees for events involving up to four variables. See (3) in Definition 23. We call it 4-consistency. But even that is not enough. Recall that Algorithm 1 uses the plug-in estimator to estimate mutual informations, based on which it runs Kruskal's Algorithm to infer the underlying tree. Naturally, we need to ensure that the estimated mutual informations are close to the actual mutual informations. However, each term in the expression for an estimated mutual information involves the appearances of estimated probabilities in the denominator (inside the logarithm), and so the value of such a term is particularly sensitive to the estimation error of a probability whose estimate appears in the denominator if this probability is small. Thus, we need an extra condition that places more stringent precision requirements on events involving up to 4 variables that have small probabilities under P. See (4) in Definition 23. We call the combination of (3) and (4) strong 4-consistency. It can be shown that strong 4-consistency holds with probability at least $1-\gamma$ for a run of Algorithm 1 (for big enough constant $B$ ), and is sufficient to guarantee the success of the algorithm. From now on we assume that $\widehat{P}$ satisfies strong 4-consistency.

The core of the argument. As in the symmetric case, the main challenge is to control the error as a result of structural mistakes (i.e. incorrectly learned edges), as opposed to "parameter mistakes" (i.e. placing the estimated pairwise distribution $\widehat{\mathrm{P}}_{i j}$ rather than the actual pairwise distribution $\mathrm{P}_{i j}$ on each edge $(i, j)$ of the tree $\widehat{T}$ of the output distribution $Q)$. So we focus on bounding the distance between $\mathrm{P}$ on one hand, and the hybrid distribution with underlying tree $\widehat{T}$ but with the correct marginal $\mathrm{P}_{i j}$ assigned to each edge $(i, j)$ of $\widehat{T}$, on the other. After getting from $T$ to $\widehat{T}$ in the sense just described, the remaining error due to the "parameter mistakes" (that is, having $\mathrm{P}_{i j}$ versus having $\widehat{\mathrm{P}}_{i j}$ on each edge $(i, j)$ of $\left.\widehat{T}\right)$ can be dealt with easily via a single application of Corollary $4 .^{3}$

As will be seen, we will go from $T$ to $\widehat{T}$ in a constant number of rounds ${ }^{4}$ where in each round we either "cut" a set of edges, "install" a set of edges, or replace a set of edges by another, resulting in a sequence of forests interpolating between $T$ and $\widehat{T}$. For each forest in the sequence, we define a hybrid distribution that is a Bayesnet with that forest as underlying graph and with $\mathrm{P}_{i j}$ assigned to each edge $(i, j)$ of the forest. This yields a sequence of hybrid distributions interpolating between $\mathrm{P}$ on one hand, and the hybrid distribution with underlying tree $\widehat{T}$ and with $\mathrm{P}_{i j}$ assigned to each edge $(i, j)$ of $\widehat{T}$, on the other. We will be able to prove that the total variation distance between any adjacent pair in this sequence of hybrid distributions is small, and the tool we will rely on to establish every such bound is, unsurprisingly, Corollary 4. The edges we "cut", "install", or swap at each of the rounds will be carefully choosen based on classifications of the edges of $T$ and $\widehat{T}$ into layers, in order to ensure that Corollary 4 is indeed applicable and that the error introduced is indeed small. This is all quite similar to the symmetric case. The rest of our discussion focuses on the realization of the program outlined in this paragraph.

As in the symmetric case, in order to obtain some guidance on how to classify the edges and define the layers (which allows us to speak more precisely about the manners in which $\widehat{T}$ might differ from $T$, and which dictates the edges we "cut", "install", or swap in

\footnotetext{
${ }^{3}$ See the passage entitled "Long hybrid arguments vs squared Hellinger subadditivity" in Section 3.1 for an explanation on why it is important to use the squared Hellinger and its subadditivity in situations like this, even though our ultimate goal is to bound the error in total variation distance.

${ }^{4}$ See the passage entitled "The challenges of going bigger" in Section 3.1 for a discussion (applicable also to the general case here) of why it is important that the number of rounds is constant.
} 
going from $T$ to $\widehat{T}$ ), as well as to bound the error in $H^{2}$ incurred by swapping edges, we need to answer (1) when can Algorithm 1 possibly make a structural mistake and (2) what is the error in $H^{2}$ if Algorithm 1 does make a structural mistake, for the modest case of $i, j, k$ lying on a path in $T$ (assuming strong 4-consistency). In this case, a structural mistake is made when Algorthm 1 prioritizes $(i, k)$ over $(i, j)$ or prioritizes $(i, k)$ over $(j, k)$. Let's focus on it prioritizing $(i, k)$ over $(i, j)$ (as the other case is symmetric), which may happen when $\widehat{\mathrm{P}}$ is such that $\mathrm{I}^{\widehat{\mathrm{P}}}\left(X_{i} ; X_{j}\right) \leq \mathrm{I}^{\widehat{\mathrm{P}}}\left(X_{i} ; X_{k}\right)$ (note that $\mathrm{I}^{\mathrm{P}}\left(X_{i} ; X_{j}\right) \geq$ $\mathrm{I}^{\mathrm{P}}\left(X_{i} ; X_{k}\right)$ by the data processing inequality).

Recall that for the symmetric case the first question above is answered by Lemma 9, which provides a necessary condition on $\alpha_{i j}$ and $\alpha_{j k}$ (i.e. $\alpha_{i j}^{2}\left(1-\left|\alpha_{j k}\right|\right) \leq \frac{\epsilon^{2}}{n}$ ) for Algorithm 2 to make the structural mistake. Combined with Lemma 10 (which says $\left.H^{2}\left(\mathrm{P}_{i j k}, \mathrm{P}_{i \widehat{j-k}}\right) \leq 2 \alpha_{i j}^{2}\left(1-\left|\alpha_{j k}\right|\right)\right)$, it yields a bound on the error in $H^{2}$ when Algorithm 2 does make the structural mistake, answering the second question above. See Lemma 11, as well as the four-node version Lemma 12.

For analyzing Algorithm 1 for the general case the following lemma is central to answering the questions above. The proof of this lemma is the only place in the entire argument where we need those implications of strong 4-consistency that are beyond those implied by just 3-consistency.

LEMMA 25. (i) If $i, j, k$ lie on a path in $T$, and $\mathrm{I}^{\widehat{P}}\left(X_{i} ; X_{j}\right) \leq$ $\mathrm{I}^{\widehat{\mathrm{P}}}\left(X_{i} ; X_{k}\right)$, then $H^{2}\left(\mathrm{P}_{i j k}, \mathrm{P}_{i \widehat{j-k}}\right) \leq 22 \frac{\epsilon^{2}}{n}$.

(ii) If $h, i, j, k$ lie on a path in $T$, and $\mathrm{I}^{\widehat{\mathrm{P}}}\left(X_{i} ; X_{j}\right) \leq \mathrm{I}^{\widehat{\mathrm{P}}}\left(X_{h} ; X_{k}\right)$, then $H^{2}\left(\mathrm{P}_{i j k}, \mathrm{P}_{i \widehat{j-k}}\right) \leq 62 \frac{\epsilon^{2}}{n}, H^{2}\left(\mathrm{P}_{h i k}, \mathrm{P}_{h-i k}\right) \leq 62 \frac{\epsilon^{2}}{n}$, and $H^{2}\left(\mathrm{P}_{h i j k}, \mathrm{P}_{h-i j-k}\right) \leq 248 \frac{\epsilon^{2}}{n}$.

Proof. (sketch of (i) showing only the main steps; for a complete proof see [6]). We expand $\mathrm{I}^{\widehat{\mathrm{P}}}\left(X_{i} ; X_{j}, X_{k}\right)$ using the chain rule for mutual information in two different ways:

$$
\mathrm{I}^{\widehat{\mathrm{P}}}\left(X_{i} ; X_{j}, X_{k}\right)=\mathrm{I}^{\widehat{\mathrm{P}}}\left(X_{i} ; X_{j}\right)+\mathrm{I}^{\widehat{\mathrm{P}}}\left(X_{i} ; X_{k} \mid X_{j}\right),
$$

and

$$
\mathrm{I}^{\widehat{\mathrm{P}}}\left(X_{i} ; X_{j}, X_{k}\right)=\mathrm{I}^{\widehat{\mathrm{P}}}\left(X_{i} ; X_{k}\right)+\mathrm{I}^{\widehat{\mathrm{P}}}\left(X_{i} ; X_{j} \mid X_{k}\right) .
$$

Comparing the two we see that $\mathrm{I}^{\widehat{\mathrm{P}}}\left(X_{i} ; X_{j}\right) \leq \mathrm{I}^{\widehat{\mathrm{P}}}\left(X_{i} ; X_{k}\right)$ implies $\mathrm{I}^{\widehat{\mathrm{P}}}\left(X_{i} ; X_{k} \mid X_{j}\right) \geq \mathrm{I}^{\widehat{\mathrm{P}}}\left(X_{i} ; X_{j} \mid X_{k}\right)$.

Notice that $i, j, k$ lying on a path in $T$ implies that $\mathrm{I}^{\mathrm{P}}\left(X_{i} ; X_{k} \mid X_{j}\right)=$ 0 . Using strong 4-consistency we can show that $\mathrm{I}^{\widehat{\mathrm{P}}}\left(X_{i} ; X_{k} \mid X_{j}\right)$ is also close to 0 . More precisely, we can show that $\mathrm{I}^{\widehat{\mathrm{P}}}\left(X_{i} ; X_{k} \mid X_{j}\right) \leq O\left(\frac{\epsilon^{2}}{n}\right)$. Thus we have $\mathrm{I}^{\widehat{\mathrm{P}}}\left(X_{i} ; X_{j} \mid X_{k}\right) \leq O\left(\frac{\epsilon^{2}}{n}\right)$.

We can show that $H^{2}\left(\widehat{\mathrm{P}}_{i j k}, \widehat{\mathrm{P}}_{i \widehat{j-k}}\right) \leq \frac{1}{2} \mathrm{I}^{\widehat{\mathrm{P}}}\left(X_{i} ; X_{j} \mid X_{k}\right)<O\left(\frac{\epsilon^{2}}{n}\right)$ (the first inequality is in fact generally true with any joint distribution taking the place of $\widehat{\mathrm{P}}$, and any $i, j, k$ not necessarily lying on a path in $T)$. So $H^{2}\left(\widehat{\mathrm{P}}_{i j k}, \widehat{\mathrm{P}}_{i \widehat{j-k}}\right) \leq O\left(\frac{\epsilon^{2}}{n}\right)$. Using strong 4-consistency we can also show that $H^{2}\left(\mathrm{P}_{i j k}, \widehat{\mathrm{P}}_{i j k}\right) \leq O\left(\frac{\epsilon^{2}}{n}\right)$ and $H^{2}\left(\widehat{\mathrm{P}}_{i \widehat{j-k}}, \mathrm{P}_{i \widehat{j-k}}\right) \leq O\left(\frac{\epsilon^{2}}{n}\right)$ (in fact just 3-consistency is enough here). Taking square roots and applying triangle inequality for Hellinger distance, we get $H^{2}\left(\mathrm{P}_{i j k}, \mathrm{P}_{i \widehat{j-k}}\right) \leq O\left(\frac{\epsilon^{2}}{n}\right)$.
Notice that Lemma 25 (i) fully answers the second question above, i.e. "what is the error in $H^{2}$ if Algorithm 1 makes a structural mistake?" The answer is $22 \frac{\epsilon^{2}}{n}$. It also partially answers the first question, i.e. "when can Algorithm 1 possibly make a structural mistake?" The answer is that, if $H^{2}\left(\mathrm{P}_{i j k}, \mathrm{P}_{i \widehat{j-k}}\right)>22 \frac{\epsilon^{2}}{n}$, then Algorithm 1 cannot possibly prioritize $(i, k)$ over $(i, j)$. We only say "partially" because we would like the answer to be stated in terms of conditions on pairwise distributions $\mathrm{P}_{i j}$ and $\mathrm{P}_{j k}$ rather than three-wise distributions, as ultimately we want to use those conditions as guidance to classify the individual edges of $\widehat{T}$ into layers. (Indeed, recall that this is what we did in the symmetric case, by utilizing Lemma 9, which provided a necessary condition for a structural mistake involving only $\alpha_{i j}$ and $\alpha_{j k}$.) Thus, we want to find conditions involving only $\mathrm{P}_{i j}$ and $\mathrm{P}_{j k}$ that would guarantee $H^{2}\left(\mathrm{P}_{i j k}, \mathrm{P}_{i j-k}\right)>22 \frac{\epsilon^{2}}{n}$. To state these conditions, we define a few useful measures on (binary) pairwise distributions. It is easier to motivate the definitions of our measures in parallel with looking for reasonable ways to define the layers via a classification of the edges of $\widehat{T}$. We proceed to do this next.

The problem of using (estimated) mutual information to classify the edges of $\widehat{T}$. Recall that, in the symmetric case, the edges of $\widehat{T}$ are classified into layers based on their $|\widehat{\alpha}|$-estimates. Indeed, the $|\widehat{\alpha}|$-estimates serve both as the edge-weights for computing the maximum weight spanning tree $\widehat{T}$ in Algorithm 2, and as the measure according to which we classify the edges of $\widehat{T}$. The situation is different in the general case: while the estimated mutual informations are used by Algorithm 1 (the Chow-Liu algorithm) as edge-weights in computing the maximum weight spanning tree $\widehat{T}$, they cannot be used for classifying the edges of $\widehat{T}$.

To see why, note that, to mimic what we did in the symmetric case, in the top layer of our layering we want to group edges of $\widehat{T}$ whose two end-nodes are almost always equal or almost always unequal (under P), as doing so will allow us to prove that within each group of the top layer it doesn't matter much in terms of $H^{2}$ whether the nodes are held together by edges of $T$ or by edges of $\widehat{T}$ (so we can swap between the two configurations without incurring too much error). More precisely, we want to identify edges whose two end-nodes are equal with probability at least $1-\Theta\left(\frac{\epsilon^{2}}{n}\right)$, or unequal with probability at least $1-\Theta\left(\frac{\epsilon^{2}}{n}\right)$. However, unlike the symmetric case in which a pair of nodes are almost always equal or almost always unequal if and only if the $|\alpha|$-value (and therefore the $|\widehat{\alpha}|$-estimate) for the pair is close to one, for the general case a pair of nodes that are almost always equal or almost always unequal could have (estimated) mutual information that lies anywhere between 0 and $\ln 2$, depending on the degree of bias of the individual node marginals. Indeed, if $X_{i}$ and $X_{j}$ are always equal, and each assumes 1 with probability $p$, then as $p$ goes from 0 to $\frac{1}{2}$ the mutual information between $X_{i}$ and $X_{j}$ goes from 0 to $\ln 2$. Thus, if we set the estimated mutual information threshold for the top layer of edges close to the higher end (say at some $\ln 2-\Theta\left(\frac{\epsilon^{2}}{n}\right)$ threshold), then we would miss edges whose end-nodes are slightly biased but are nevertheless almost always equal or almost always unequal to each other. On the other hand, if we set the estimated mutual information threshold for the top layer at any value bounded away from the higher end, 
then we may include, for example, edges whose two end-nodes are (close to) uniform but are nevertheless neither almost always equal nor almost always unequal, in the sense we described above.

The above discussion serves to motivate the use of different measures than mutual information for classifying the edges of $\widehat{T}$. Those new measures - minmrg, mindiag, mindisc, and $\boldsymbol{I}_{\boldsymbol{H}^{2}}-$ are defined below along with the layering.

The top layer. For the top layer we use a combination of minmrg and mindiag, and classify an edge $(i, j)$ of $\widehat{T}$ as a $\widehat{T}$-avenue (we will explain later why we don't call it a "road" reflecting our terminology for the symmetric case) if minmrg $\left(\widehat{\mathrm{P}}_{i j}\right) \geq 10^{6} \frac{\epsilon^{2}}{n}$ and mindiag $\left(\widehat{\mathrm{P}}_{i j}\right) \leq$ $10^{5} \frac{\epsilon^{2}}{n},{ }^{5}$ where

$$
\begin{aligned}
& \operatorname{minmrg}\left(\widehat{\mathrm{P}}_{i j}\right)=\min \left\{\widehat{\mathrm{P}}_{i}(1), \widehat{\mathrm{P}}_{i}(-1), \widehat{\mathrm{P}}_{j}(1), \widehat{\mathrm{P}}_{j}(-1)\right\}, \text { and } \\
& \operatorname{mindiag}\left(\widehat{\mathrm{P}}_{i j}\right)=\min \left\{\widehat{\mathrm{P}}_{i j}\left(X_{i}=X_{j}\right), \widehat{\mathrm{P}}_{i j}\left(X_{i}=-X_{j}\right)\right\} .
\end{aligned}
$$

The $\widehat{T}$-avenues cluster the nodes into connected components, each of which is called a broken-city, a term we explain later.

Striving to make the broken-cities T-connected. The condition mindiag $\left(\widehat{\mathrm{P}}_{i j}\right) \leq 10^{5} \frac{\epsilon^{2}}{n}$ obviously says that $X_{i}$ and $X_{j}$ are estimated to be almost always equal or almost always unequal. But why do we need the condition minmrg $\left(\widehat{\mathrm{P}}_{i j}\right) \geq 10^{6} \frac{\epsilon^{2}}{n}$ ? Recall that in the symmetric case each city is shown to be not only $\widehat{T}$-connected, but also $T$-connected, which allows us to apply Corollary 4 with $A_{1}, \ldots, A_{\Lambda}$ being the cities in order to bound $H\left(\mathrm{P}^{(2)}, \mathrm{P}^{(3)}\right)$ (the pair of distributions in our final hybrid argument related by the replacement of $T$-highways by the $\widehat{T}$-highways; see Section 3 ). We hope the same is true in the general case: if $i$ and $j$ belong to the same broken-city, we would hope that all the nodes on the path in $T$ between $i$ and $j$ also belong to that broken-city. If we didn't impose the condition $\operatorname{minmrg}\left(\widehat{\mathrm{P}}_{i j}\right) \geq 10^{6} \frac{\epsilon^{2}}{n}$ in our definition of $\widehat{T}$-avenues, then we can't exclude, for example, that $X_{i}$ and $X_{j}$ are both always equal to 1 . In this extreme case, the distributions for the intermediate nodes and edges on the path in $T$ between $i$ and $j$ can be arbitrary, and it would be impossible to prove that each intermediate node also belongs to the broken-city containing $i$ and $j$. The main issue here is that even though $X_{i}$ and $X_{j}$ are always equal, they are also independent of each other! This phenomenon can only occur when the individual marginals are allowed to be very biased, which can happen in the general case, but not in the symmetric case. In general, to avoid this kind of situations, we need to prevent including in the top layer edges of $\widehat{T}$ whose end-nodes are almost always equal or almost always unequal but are also almost independent. To do that, we have to exclude edges of $\widehat{T}$ whose end-nodes are "too biased." The condition minmrg $\left(\widehat{\mathrm{P}}_{i j}\right) \geq 10^{6} \frac{\epsilon^{2}}{n}$ does exactly that.

With a lower bound on minmrg included in the requirement for a $\widehat{T}$-avenue, the following becomes applicable, as we will see.

LeMmA 26. If $i, j, k$ lie on a path in $T$, and $\operatorname{minmrg}\left(\mathrm{P}_{i k}\right)>8$. $\operatorname{mindiag}\left(\mathrm{P}_{i k}\right)$, then

(i) $\operatorname{minmrg}\left(\mathrm{P}_{j}\right) \geq \operatorname{minmrg}\left(\mathrm{P}_{i k}\right)-\operatorname{mindiag}\left(\mathrm{P}_{i k}\right)$;

(ii) $\max \left\{\operatorname{mindiag}\left(\mathrm{P}_{i j}\right), \operatorname{mindiag}\left(\mathrm{P}_{j k}\right)\right\} \leq \operatorname{mindiag}\left(\mathrm{P}_{i k}\right)$;

\footnotetext{
${ }^{5}$ Again, we could have let $T$ dictate the layering, or based our classification on $\mathrm{P}$ instead of $\widehat{\mathrm{P}}$, or both. See footnote 2 in Section 3.1 for a discussion of our choice.
}

(iii) $\operatorname{mindiag}\left(\mathrm{P}_{i j}\right)+\operatorname{mindiag}\left(\mathrm{P}_{j k}\right) \leq \frac{7}{6} \min \operatorname{diag}\left(\mathrm{P}_{i k}\right)$.

Lemma 26 is in some sense an analog of the multiplicativity of $\alpha$-values along a path in $T$, for the measure mindiag (recall that the $\alpha$-values provide a measure on pairwise distributions for the symmetric case). Roughly speaking, Lemma 26 (ii) says that closer pairs along a path in $T$ are stronger as measured by mindiag (for $\alpha$-values for the symmetric case the analogous relationship is $\min \left\{\left|\alpha_{i j}\right|,\left|\alpha_{j k}\right|\right\} \geq\left|\alpha_{i k}\right|$ ), and (iii) controls the manner in which the strengths of the pairs (as measured by mindiag) concatenate/decompose along a path in $T$.

Note that Lemma 26 (iii) implies that if $(i, k)$ is a $\widehat{T}$-avenue and $j$ is on the path in $T$ between $i$ and $k$ then at least one of mindiag $\left(\mathrm{P}_{i j}\right)$ and mindiag $\left(\mathrm{P}_{j k}\right)$ is significantly less than mindiag $\left(\mathrm{P}_{i k}\right)$ (it is important that we have "significantly less than" instead of just "less than" here because we need to allow space for estimation error). Say it is mindiag $\left(\mathrm{P}_{i j}\right)$. Then following some quite involved argument (the main complication being that $(i, j)$ might not be an edge of $\widehat{T}$ so we can't simply deduce that $i$ and $j$ belong to the same broken-city even if $\widehat{\mathrm{P}}_{i j}$ satisfies the criteria for a $\widehat{T}$-avenue; instead, we have to reason about the edges of $\widehat{T}$ on the path in $\widehat{T}$ between $i$ and $j$; see [6]), we can arrive at the desired conclusion that $j$ belongs to the same broken-city as $i$ does, as long as $\operatorname{minmrg}\left(\mathrm{P}_{j}\right) \geq 10^{7} \frac{\epsilon^{2}}{n}$. We call a node $j$ biased if $\operatorname{minmrg}\left(\mathrm{P}_{j}\right)<10^{7} \frac{\epsilon^{2}}{n}$. Consequently, every unbiased node on the path in $T$ between the two end-nodes of some $\widehat{T}$-avenue must belong to the same broken-city as those two endnodes. In reality, the constant $10^{7}$ in the threshold for biased nodes can be replaced by any number strictly greater than $10^{6}$ (recall $10^{6}$ is the constant in the minmrg threshold for $\widehat{T}$-avenues) as long as we make $B$ (in the sample complexity of the algorithm) big enough.

Failure to make the broken-cities T-connected. Unfortunately, the above argumentation still doesn't yield that every broken-city is $T$-connected due to potential "holes" created by biased nodes (a "hole" $j$ for a broken-city is a node which doesn't belong to the broken-city, but which lies on the path in $T$ between some $i$ and $k$ belonging to the broken-city). Indeed, our analysis is ultimately not going to guarantee that every broken-city is necessarily $T$ connected. Whatever we set the minmrg threshold for $\widehat{T}$-avenues to be, the minmrg threshold for a node to be considered unbiased has to be strictly greater than that in order for us to be able to prove that unbiased nodes won't create any "holes." On the other hand, if $i, j, k$ lie on a path in $T$, we can't eliminate the possibility that $\operatorname{minmrg}\left(\mathrm{P}_{j}\right)$ is strictly less than both $\operatorname{minmrg}\left(\mathrm{P}_{i}\right)$ and $\operatorname{minmrg}\left(\mathrm{P}_{k}\right)$ (or their $\widehat{\mathrm{P}}$ counterparts) (see Lemma 26 (i) for the best lower bound we can get). Consequently, it could potentially happen that $(i, k)$ is classified as a $\widehat{T}$-avenue, but $j$ is biased. When that happens, we can't prove that $j$ belongs to the same broken-city as $i$ and $k$ do. So a broken-city might not be $T$-connected (which is why we don't just call it a "city"). We have to work around that.

The remedy. The key to the remedy is, as we have already noted, that every "hole" must be biased. That is, every "hole" is either almost always equal to 1 , or almost always equal to -1 , under P. Roughly speaking, the "holes" can be neglected, because every edge incidental to a "hole" must be close to being independent in $H^{2}$ under $\mathrm{P}$ due to the "hole" having a small minmrg under $\mathrm{P}$ 
(see [6]). We define the convex hull (in $T$ ) of a broken-city by filling in all the "holes" (see [6]). While any two different broken-cities must be disjoint, their convex hulls might intersect. Whenever the convex hulls of two broken-cities intersect, we have to merge those broken-cities. The resulting clusters after we merge every pair of broken-cities whose convex hulls intersect are called cities. It can be shown that every city is $T$-connected (see [6]). While a city might not be $\widehat{T}$-connected, we will be able to work around that.

To complete our definitions at the top layer, we classify every edge of $T$ whose two end-nodes belong to the same city as a $T$-road. Recall that in the symmetric case, one of the steps in the process of going from $T$ to $\widehat{T}$ is to replace the set of all $T$-roads by the set of all $\widehat{T}$-roads, and that each city is spanned by the $T$-roads in it, as well as by the $\widehat{T}$-roads in it. We would like something similar for the general case to make our argument down the road simpler and clearer. Because each city $C$ is $T$-connected, it is spanned by those $T$-roads whose two end-nodes belong to $C$. On the other hand, the $\widehat{T}$-avenues whose two end-nodes belong to $C$ are not necessarily enough to span $C$ (which is why we don't call them " $\widehat{T}$-roads"); they only span the broken-cities contained in $C$ individually. To augment them into a spanning set for $C$ we have to throw in some edges that connect together the broken-cities contained in $C$. In fact, those additional edges can be selected from among the set of $T$-roads with end-nodes in $C$. See [6] for details. We call each of those additional edges a $T$-trail. Thus, each city $C$ is spanned by the union of the $\widehat{T}$-avenues with end-nodes in $C$ and the $T$-trails with end-nodes in $C$. One of the steps in the process of going from $T$ to $\widehat{T}$ is to replace the set of all $T$-roads by the union of the set of all $\widehat{T}$-avenues and the set of all $T$-trails. Notice that the $T$-trails are selected from among the $T$-roads, and so this round of edge replacement is in fact leaving those edges unchanged. Again, it is for the clarity of our argument that we insist on their involvement so that within each city we are replacing one spanning tree by another spanning tree (see the bounding argument for $H\left(\mathrm{P}^{(3)}, \mathrm{P}^{(4)}\right)$ in [6]). Of course, the $T$-trails are not necessarily edges of $\widehat{T}$ so we have to get rid of them eventually. It can be shown that every $T$-trail must be incident to at least one biased node, and so it is close to being independent in $H^{2}$ under P. Consequently, they can be cut away without incurring too much error in $H^{2}$ (see the bounding argument for $H\left(\mathrm{P}^{(4)}, \mathrm{P}^{(5)}\right)$ in [6]).

For a diagrammatic illustration of the top layer see Figure 5. The solid connections represent edges of $T$, and the dashed connections represent edges of $\widehat{T}$. The small shaded circles represent the broken-cities, the shaded regions with irregular curved boundaries represent the convex hulls in $T$ of the broken-cities, and the big hollow circle represents the city $C$. The operations of taking convex hulls in $T$ fill in the "holes." For example, $16 \in \operatorname{conv}_{T}\left(\widetilde{C}_{5}\right)$ because the node 16 lies on the path in $T$ between nodes 12 and 13, both of which belong to $\widetilde{C}_{5}$. Recall that all "holes" must be biased (that is, having minmrg less than $10^{7} \frac{\epsilon^{2}}{n}$ ). In our case, the "holes" are marked with cross marks right next to them. Also, the broken-cities whose convex hulls in $T$ intersect are merged into the same city. In our case, the broken-cities $\widetilde{C}_{1}, \ldots, \widetilde{C}_{7}$ are merged into the city $C$, while $\widetilde{C}_{8}$ and $\widetilde{C}_{9}$ belong to some other city (not shown) because their convex hulls in $T$ don't intersect with the convex hull in $T$ of any of $\widetilde{C}_{1}, \ldots, \widetilde{C}_{7}$. Lastly, we use tiny hollow circles placed at the middle of the edges to mark the six $T$-roads that, along with the $\widehat{T}$-avenues in $C$, form a spanning tree of $C$. We can select those $T$-roads to be the $T$-trails in $C$ (the selection is not unique: we could have selected $(13,17)$ instead of $(13,16)$, for example).

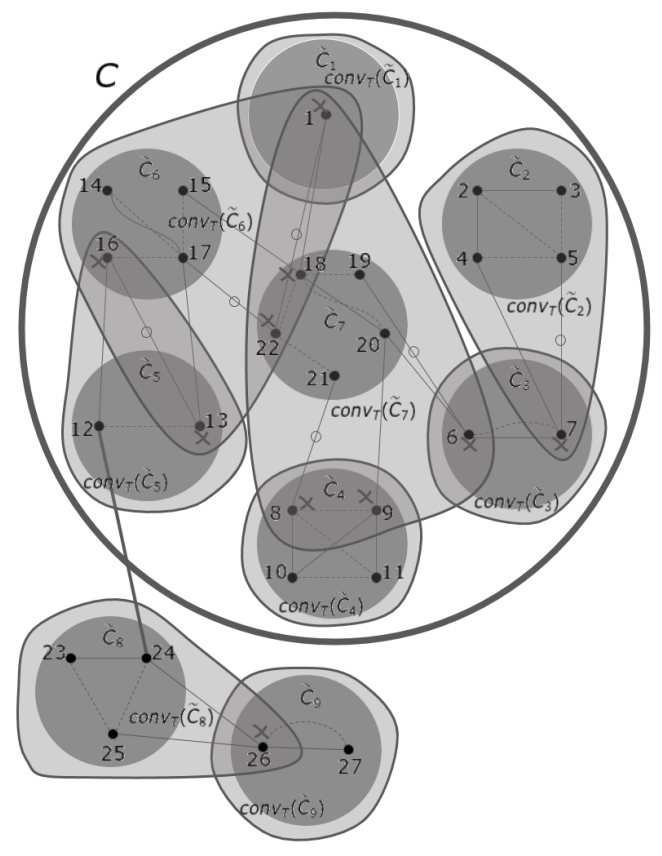

Figure 5: A diagrammatic illustration of our hierarchy. For a description of the figure see the last paragraph of "The remedy" in Section 4.1.

The second layer. For the next layer, we classify an edge $(i, j)$ of $\widehat{T}$ with minmrg $\left(\widehat{\mathrm{P}}_{i j}\right) \geq 10^{8} \frac{\epsilon^{2}}{n}$ and mindisc $\left(\widehat{\mathrm{P}}_{i j}\right) \geq \frac{1}{2}$ that is not a $\widehat{T}$-avenue as a $\widehat{T}$-highway, where

$$
\begin{aligned}
& \operatorname{mindisc}\left(\widehat{\mathrm{P}}_{i j}\right) \\
& =\min \left\{\left|\widehat{\mathrm{P}}_{i \mid j}(1 \mid 1)-\widehat{\mathrm{P}}_{i \mid j}(1 \mid-1)\right|,\left|\widehat{\mathrm{P}}_{j \mid i}(1 \mid 1)-\widehat{\mathrm{P}}_{j \mid i}(1 \mid-1)\right|\right\} .
\end{aligned}
$$

The $\widehat{T}$-highways link the broken-cities into broken-countries, and link the cities into countries (from which it follows immediately that each country is a union of broken-countries). An edge $(i, j)$ of $T$ with $i, j$ belonging to different cities in the same country is classified as a $T$-highway. We prove the following structural results (note that (iii) is an analog of Lemma 16 for the symmetric case):

LEMMA 27. (i) A $\widehat{T}$-highway goes between different cities.

(ii) There can be at most one $\widehat{T}$-highway and at most one $T$ highway between any pair of cities.

(iii) There is a $\widehat{T}$-highway between a pair of cities if and only if there is a T-highway between the same pair of cities, and if so, those two highways in fact go between the same pair of broken-cities.

Lemma 28. If $i, j, k$ lie on a path in $T$, then $H^{2}\left(\mathrm{P}_{i j k}, \mathrm{P}_{i \widehat{j-k}}\right) \geq$ $\frac{1}{100} \operatorname{mindisc}\left(\mathrm{P}_{i j}\right)^{2} \cdot \min \left\{\operatorname{minmrg}\left(\mathrm{P}_{j}\right), \min \operatorname{diag}\left(\mathrm{P}_{j k}\right)\right\}$. 
As in the symmetric case, we will replace the set of all $T$-highways with the set of all $\widehat{T}$-highways in a single round of edge replacements in the process of going from $T$ to $\widehat{T}$ (see the bounding argument for $H\left(\mathrm{P}^{(2)}, \mathrm{P}^{(3)}\right)$ in [6]). Also note that Lemma 27 (iii) and the fact that every city is $T$-connected imply that every country is $T$-connected.

The third layer. For the next layer, we classify an edge $(i, j)$ of $\widehat{T}$ with $I_{H^{2}}\left(\widehat{\mathrm{P}}_{i j}\right) \geq 10^{10} \frac{\epsilon^{2}}{n}$ that is not a $\widehat{T}$-avenue or a $\widehat{T}$-highway as a $\widehat{T}$-railway, where $I_{H^{2}}\left(\widehat{\mathrm{P}}_{i j}\right)=H^{2}\left(\widehat{\mathrm{P}}_{i j}, \widehat{\mathrm{P}}_{i j}^{(\mathrm{ind})}\right)$, and $\widehat{\mathrm{P}}_{i j}^{(\mathrm{ind})}$ is the independent pairwise distribution that has the same individual node marginals at $i$ and $j$, respectively, as $\widehat{\mathrm{P}}_{i j}$ does. The $\widehat{T}$-railways link the broken-countries into broken-continents, and link the countries into continents (from which it follows immediately that each continent is a union of broken-continents). An edge $(i, j)$ of $T$ with $i, j$ belonging to different countries in the same continent is classified as a $\boldsymbol{T}$-railway. We prove the following structural results (note that (iii) is an analog of Lemma 19 for the symmetric case):

LEMMA 29. (i) $A \widehat{T}$-railway goes between different countries.

(ii) There can be at most one $\widehat{T}$-railway and at most one T-railway between any pair of countries.

(iii) There is a $\widehat{T}$-railway between a pair of countries if and only if there is a T-railway between the same pair of countries, and if so, those two railways in fact go between the same pair of broken-countries.

Lemma 30. If $i, j$, $k$ lie on a path in $T$, then $H^{2}\left(\mathrm{P}_{i j k}, \mathrm{P}_{i \widehat{j-k}}\right) \geq$ $\frac{1}{100} I_{H^{2}}\left(\mathrm{P}_{i j}\right) \cdot\left(1-\operatorname{mindisc}\left(\mathrm{P}_{j k}\right)\right)^{2}$.

As in the symmetric case, we replace the set of all $T$-railways with the set of all $\widehat{T}$-railways in a single round of edge replacements in the process of going from $T$ to $\widehat{T}$ (see the bounding of $H\left(\mathrm{P}^{(1)}, \mathrm{P}^{(2)}\right)$ in [6]). Also note that Lemma 29 (iii) and the fact that every country is $T$-connected imply that every continent is $T$-connected.

The bottom layer. Finally, an edge $(i, j)$ of $\widehat{T}$ that is yet to be classfied is classified as a $\widehat{T}$-tunnel, and an edge $(i, j)$ of $T$ that is yet to be classified is classified as a $T$-airway. While a $T$-airway must go between two different continents, a $\widehat{T}$-tunnel might not. In fact, the two end-nodes of a $\widehat{T}$-tunnel might belong to the same city, to different cities in the same country, to different countries in the same continent, or to different continents (that's why we call it a $\widehat{T}$-tunnel, instead of a " $\widehat{T}$-airway"). Nevertheless, it can be shown that an edge that is a $\widehat{T}$-tunnel or $T$-airway must be close to being independent in $H^{2}$ under $\mathrm{P}$, so that they can be cut away without incurring too much error in $H^{2}$ (see the bounding argument for $H\left(\mathrm{P}, \mathrm{P}^{(1)}\right)$ and $H\left(\mathrm{P}^{(6)}, \mathrm{P}^{(7)}\right)$ in [6]).

Summary of layering. To summarize, the edges of $\widehat{T}$ are classified into $\widehat{T}$-avenues, $\widehat{T}$-highways, $\widehat{T}$-railways, and $\widehat{T}$-tunnels based on a combination of the measures minmrg, mindiag, mindisc, and $I_{H^{2}}$. The $n$ nodes are clustered into broken-cities by the $\widehat{T}$-avenues, which are further clustered into broken-countries by the $\widehat{T}$-highways, which are further clustered into broken-continents by the $\widehat{T}$-railways, which are finally linked into one component by the $\widehat{T}$-tunnels.

Note that each broken-city, broken-country, or broken-continent is $\widehat{T}$-connected by definition. Unlike what happened in the symmetric case, it is however not necessarily $T$-connected. To remedy that, we define the convex hull (in $T$ ) of a broken-city by filling in all the "holes" (a "hole" for a broken-city is a node which doesn't belong to the broken-city, but which lies on the path in $T$ between some two nodes that belong to the broken-city; we can prove that every "hole" is biased, in that it is almost always 1 or almost always $-1)$. Whenever the convex hulls of two broken-cities intersect, we merge those broken-cities together. The resulting clusters after we merge every such pair of broken-cities are called the cities. The $\widehat{T}$-highways (each of which can be shown to go between different cities) link the cities into countries, and the $\widehat{T}$-railways (each of which can be shown to go between different countries) link the countries into continents. This hierarchical classification of nodes into groups then induces a classification of the edges of $T$ : an edge of $T$ is classified as a $T$-road if its two end-nodes belong to the same city, a $T$-highway if its two end-nodes belong to different cities in the same country, a $T$-railway if its two end-nodes belong to different countries in the same continent, or a $T$-airway if its two end-nodes belong to different continents. For edges of $\widehat{T}$, we similarly have that the two end-nodes of each $\widehat{T}$-avenue belong to the same (broken-)city, those of each $\widehat{T}$-highway belong to different (broken-)cities in the same (broken-)country, and those of each $\widehat{T}$-railway belong to different (broken-)countries in the same (broken-)continent. However, while the two end-nodes of each $\widehat{T}$ tunnel surely belong to different broken-continents, they might in fact belong to the same city, different cities in the same country, different countries in the same continent, or different continents.

We prove that every city, country, or continent is $T$-connected. (They are not necessarily $\widehat{T}$-connected, though.) Furthermore, we prove that the $T$-highways and $\widehat{T}$-highways can be matched into parallel pairs, i.e. there is a $\widehat{T}$-highway between two broken-cities if and only if there is a $T$-highway between them (and there is at most one such pair between every pair of cities). The same goes for railways: there is a $\widehat{T}$-railway between two broken-countries if and only if there is a $T$-railway between them (and there is at most one such pair between every pair of countries). A consequence of our statements is that the exact same cities, countries, and continents would have been achieved if we were to cluster the nodes first by $T$-roads, then $T$-highways, then $T$-railways, and finally $T$-airways.

Lastly, for every city $C$ we select among the set of $T$-roads with end-nodes in $C$ a subset that, together with the $\widehat{T}$-avenues with end-nodes in $C$, form a spanning tree of $C$. We call those selected edges $T$-trails. We can prove that every $T$-trail must be incidental to a biased node and is therefore close to being independent in $H^{2}$.

For a diagrammatic illustration of our structural results see Figure 6. The solid connections represent edges of $T$, and the dashed connections represent edges of $\widehat{T}$. The thickness of the line increases as we go down in the hierarchy, with the $T$-roads and $\widehat{T}$-avenues being the thinnest and the $T$-airways and $\widehat{T}$-tunnels being the thickest. The shaded circles with dashed boundaries represent the brokencities, the shaded rectangular boxes with dashed boundaries represent the broken-countries, and the shaded regions with dashed saw-tooth boundaries represent the broken-continents. The hollow circular or oval regions with solid boundaries represent the cities, the hollow regions with solid axis-aligned boundaries represent the countries, and the hollow regions with solid saw-tooth boundaries represent the continents. To avoid cluttering the figure, we don't 
show the convex hulls (in $T$ ) of the broken-cities. Note that the $T$-highways and $\widehat{T}$-highways can be matched into parallel pairs, each going between the same pair of broken-cities (in different cities in the same country). An example is $(1,2)$ and $(3,4)$. Similarly, the $T$-railways and $\widehat{T}$-railways can be matched into parallel pairs, each going between the same pair of broken-countries (in different countries in the same continent). An example is $(5,6)$ and $(7,8)$. This parallelism needs not hold other types of edges. For example, there is no edge of $\widehat{T}$ that goes between the same pair of continents as the $T$-airway $(9,10)$ does, and there is no $T$-airway that goes between the same pair of continents as the $\widehat{T}$-tunnel $(11,12)$ does. The $\widehat{T}$-tunnels can behave wildly, in the sense that the two end-nodes of a $\widehat{T}$-tunnel could belong to different continents (e.g. $(13,14))$, to different countries in the same continent (e.g. $(15,16))$, to different cities in the same country (e.g. $(17,18))$, or even to the same city (e.g. $(19,20)$ ). Lastly, we use tiny hollow circles placed at the middle of the edges to mark one possible selection for the $T$-trails. (There are some structural properties illustrated in the diagram that were not discussed in the outline above. For example, the path in $T$ between the end-nodes of a $\widehat{T}$-highway or a $\widehat{T}$-railway must not contain any biased node. Consequently, it must not contain any "hole" for any of the broken-cities. Those properties are used in the proof.)

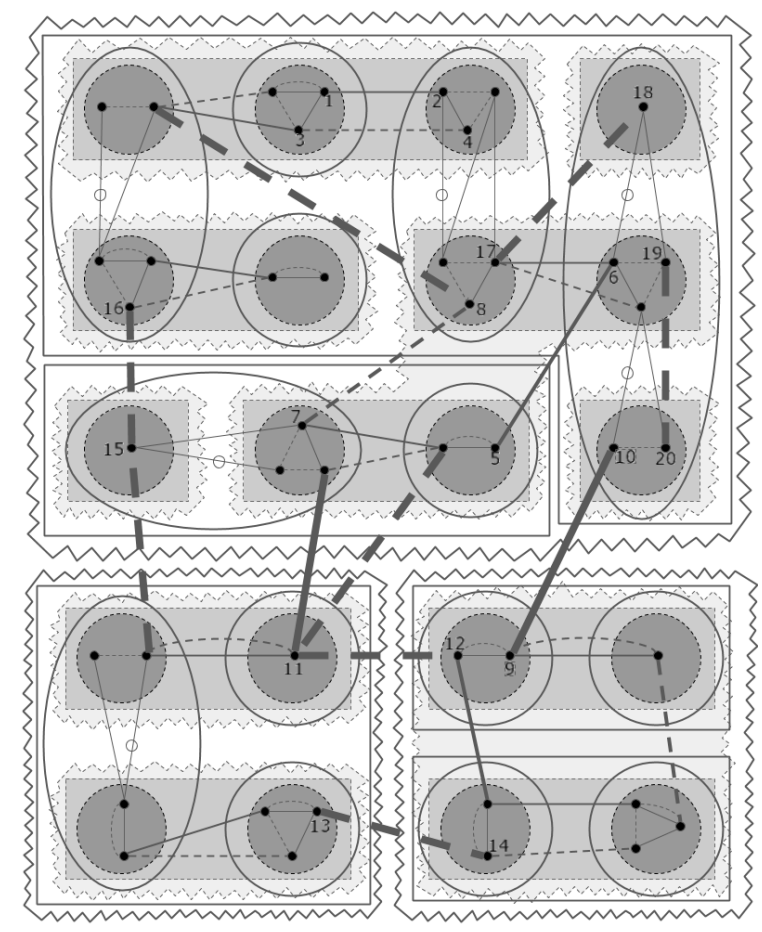

Figure 6: A diagrammatic illustration of our hierarchy. For a description of the figure see the last paragraph of "Summary of layering" in Section 4.1.

Going from $T$ to $\widehat{T}$ for the general case. To go from $T$ to $\widehat{T}$, we first cut away all the $T$-airways, then replace the set of $T$-railways by the set of $\widehat{T}$-railways, then replace the set of $T$-highways by the set of $\widehat{T}$-highways, then replace the set of $T$-roads by the union of the set of $\widehat{T}$-avenues and the set of $T$-trails, then cut away all the $T$-trails, and finally install all the $\widehat{T}$-tunnels. See the complete version [6] for a rigorous treatment of the bounding of $d_{\mathrm{TV}}(\mathrm{P}, \mathrm{Q})$.

\section{ACKNOWLEDGEMENTS}

This work was supported by NSF Awards IIS-1741137, CCF-1617730 and CCF-1901292, by a Simons Investigator Award, by the DOE PhILMs project (No. DE-AC05-76RL01830). Pan has no current affiliation to MIT. This manuscript was submitted for review in November 2020. It was finalized for publication by Daskalakis alone.

\section{REFERENCES}

[1] Guy Bresler. 2015. Efficiently learning Ising models on arbitrary graphs. In Proceedings of the forty-seventh annual ACM Symposium on Theory Of Computing (STOC).

[2] C Chow and Cong Liu. 1968. Approximating discrete probability distributions with dependence trees. IEEE transactions on Information Theory 14, 3 (1968), 462-467.

[3] C Chow and T Wagner. 1973. Consistency of an estimate of tree-dependent probability distributions. IEEE Transactions on Information Theory 19, 3 (1973), 369-371.

[4] Constantinos Daskalakis, Nishanth Dikkala, and Gautam Kamath. 2019. Testing Ising Models. IEEE Trans. Inf. Theory 65, 11 (2019), 6829-6852. https://doi.org/ 10.1109/TIT.2019.2932255

[5] Constantinos Daskalakis and Qinxuan Pan. 2017. Square Hellinger Subadditivity for Bayesian Networks and its Applications to Identity Testing. In the 30th Conference on Learning Theory (COLT).

[6] Constantinos Daskalakis and Qinxuan Pan. 2020. Sample-Optimal and Efficient Learning of Tree Ising models. CoRR abs/2010.14864 (2020). arXiv:2010.14864 https://arxiv.org/abs/2010.14864

[7] Luc Devroye, Abbas Mehrabian, and Tommy Reddad. 2019. The minimax learning rate of normal and Ising undirected graphical models. Electronic fournal of Statistics (2019).

[8] Linus Hamilton, Frederic Koehler, and Ankur Moitra. 2017. Information theoretic properties of Markov random fields, and their algorithmic applications. In Advances in Neural Information Processing Systems. 2463-2472.

[9] Ali Jalali, Pradeep Ravikumar, Vishvas Vasuki, and Sujay Sanghavi. 2011. On learning discrete graphical models using group-sparse regularization. In Proceedings of the fourteenth international conference on artificial intelligence and statistics. 378-387.

[10] Adam Klivans and Raghu Meka. 2017. Learning graphical models using multiplicative weights. In Proceedings of the forty-ninth annual ACM Symposium on Theory Of Computing (STOC).

[11] Frederic Koehler. 2020. A Note on TV Learning of Tree Models. Personal Communication, http://math.mit.edu/ fkoehler/tv_note.pdf.

[12] Steffen L Lauritzen. 1996. Graphical models. Vol. 17. Clarendon Press

[13] Mukund Narasimhan and Jeff A. Bilmes. 2004. PAC-learning Bounded Tree-width Graphical Models. In the 20th Conference in Uncertainty in Artificial Intelligence (UAI).

[14] Judea Pearl. 2014. Probabilistic reasoning in intelligent systems: networks of plausible inference. Elsevier.

[15] Pradeep Ravikumar, Martin J Wainwright, and John D Lafferty. 2010. Highdimensional Ising model selection using $\ell_{1}$-regularized logistic regression. The Annals of Statistics 38, 3 (2010), 1287-1319.

[16] Narayana P. Santhanam and Martin J. Wainwright. 2012. Information-Theoretic Limits of Selecting Binary Graphical Models in High Dimensions. IEEE Trans. Information Theory 58, 7 (2012), 4117-4134.

[17] Vincent Y. F. Tan, Animashree Anandkumar, Lang Tong, and Alan S. Willsky. 2011. A Large-Deviation Analysis of the Maximum-Likelihood Learning of Markov Tree Structures. IEEE Trans. Information Theory 57, 3 (2011), 1714-1735.

[18] Marc Vuffray, Sidhant Misra, Andrey Lokhov, and Michael Chertkov. 2016. Interaction screening: Efficient and sample-optimal learning of Ising models. In Advances in Neural Information Processing Systems. 2595-2603.

[19] Marc Vuffray, Sidhant Misra, and Andrey Y. Lokhov. 2019. Efficient Learning of Discrete Graphical Models. arXiv:1902.00600 [cs.LG]

[20] Martin J Wainwright, Michael I Jordan, et al. 2008. Graphical models, exponential families, and variational inference. Foundations and Trends ${ }^{\circledR}$ in Machine Learning 1, 1-2 (2008), 1-305.

[21] Shanshan Wu, Sujay Sanghavi, and Alexandros G. Dimakis. 2019. Sparse Logistic Regression Learns All Discrete Pairwise Graphical Models. In the 32nd Annual Conference on Neural Information Processing Systems. 